Original paper

\title{
Origin of the pegmatite veins within the skarn body at Vevčice near Znojmo (Gföhl Unit, Moldanubian Zone)
}

\author{
David BURIÁNEK ${ }^{*}$, Stanislav HOUZAR², Lukáš KRMÍČEK ${ }^{3,4}$, Jaroslav ŠMERDA ${ }^{5}$ \\ ${ }^{1}$ Czech Geological Survey, Leitnerova 22, 65859 Brno, Czech Republic; david.burianek@geology.cz \\ ${ }^{2}$ Department of Mineralogy and Petrography, Moravian Museum, Zelný trh 6, 67401 Brno, Czech Republic \\ ${ }^{3}$ Faculty of Civil Engineering, AdMaS Centre, Brno University of Technology, Veveři 95, 60200 Brno, Czech Republic \\ ${ }^{4}$ Institute of Geology of the Czech Academy of Sciences, v. v. i., Rozvojová 269, 16502 Prague 6, Czech Republic \\ ${ }^{5}$ South Moravian Museum in Znojmo, Přemyslovců 8/129, 66945 Znojmo, Czech Republic \\ * Corresponding author
}

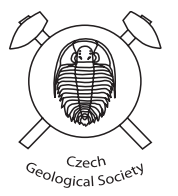

The lower crustal Gföhl Unit (Moldanubian Zone of the Bohemian Massif) consists of various felsic orthogneisses to migmatites (the so-called Gföhl gneiss) that are commonly associated with the high-pressure felsic granulites, serpentinized garnet/spinel peridotites, garnet pyroxenites, eclogites and skarns. The relatively small skarn bodies at Vevčice in southern Moravia have the prevailing assemblage of medium- to coarse-grained granoblastic clinopyroxene + garnet \pm amphibole \pm epidote. The skarn is cut by narrow veins of diorite pegmatite $\left(\mathrm{SiO}_{2}=46-56 \mathrm{wt}\right.$. \%) which is geochemically distinct from granitic melt generated by migmatization of surrounding orthogneisses. Major pegmatite minerals are plagioclase $\left(\mathrm{An}_{15-44}\right)$, amphibole (K-rich hastingsite and potassic-hastingsite) and quartz, accompanied by minor K-feldspar, garnet, allanite-epidote, clinopyroxene, and accessory titanite. The pegmatites are characterized by high contents of $\mathrm{Fe}_{2} \mathrm{O}_{3 \text { tot }}\left(11.2-17.5\right.$ wt. \%), $\mathrm{CaO}$ (7.5-10.1 wt. \%), $\mathrm{Na}_{2} \mathrm{O}$ (2.9-3.3 wt. \%) and low contents of MgO (1.4-1.6 wt. \%), $\mathrm{K}_{2} \mathrm{O}$ (1.2-1.6 wt. \%), Rb (43-86 ppm). Whole-rock and mineral chemistry of diorite pegmatites are consistent with their origin as a product of melt infiltration from surrounding migmatites and subsequent contamination by the country-rock skarn, with or without, fractional crystallization/crystal accumulation. Similarity in composition of amphiboles in the skarn and in the pegmatite can indicate formation under similar conditions, at $c .750{ }^{\circ} \mathrm{C}$ and $0.7-0.8 \mathrm{GPa}$.

Keywords: diorite pegmatite, skarn, mineralogy, geochemistry, Moldanubian Zone, Bohemian Massif

Received: 13 March, 2016; accepted: 7 March, 2017; handling editor: V. Kachlik

\section{Introduction}

Regionally metamorphosed skarns of the Moldanubian Zone of the Bohemian Massif are commonly intersected by numerous veins and pockets of coarse-grained pegmatites of diorite to tonalite composition. They are known as contaminated amphibole-rich pegmatite (Němec 1963a, 1968), plagioclase pegmatite (Staněk 1977), contaminated primitive amphibole-fluorite-allanite pegmatite (Novák 2005) or amphibole-bearing pegmatite (Novák et al. 2013). These rocks form irregular to discordant veins which frequently lack modal zoning and disequilibrium textures typical of pegmatites (Novák et al. 2013). These veins are considered to be products of melt reaction between external anatectic melts and the host skarns; reaction zones formed by alkali-rich calcic amphiboles and biotite are often developed along the contacts with the host skarn (Slavík 1951; Němec 1963a, 1991; Novák 2005; Žáček 2007; Novák et al. 2013). Excluding the occurrences at Vlastějovice and Líšná, those veins were not studied in detail; their mineralogy, chemical composition and origin remain essentially unknown.
The newly discovered skarn at Vevčice near Znojmo, Czech Republic, represents one of few outcrops suitable for detailed study of the relationship between skarn and amphibole-rich diorite pegmatites. The several-cm thick pegmatite veins consist of variable amounts of amphibole and plagioclase that considerably prevail over K-feldspar and quartz (both up to 5 vol. \%). Minor epidote or garnet is locally present. The aim of this study is to decipher relations between mineral assemblage of the skarn and pegmatite and to discuss the origin of the latter.

\section{Geological background}

\subsection{Geological setting}

The Moldanubian Zone, the high-grade metamorphosed core of the Bohemian Massif, represents a crustal and upper-mantle tectonic collage assembled during the Variscan Orogeny and modified by several events of superimposed deformation and high- to low-grade metamorphic overprint (Fiala et al. 1995; Schulmann et al. 2008). Two principal tectonic/lithological assemblages 


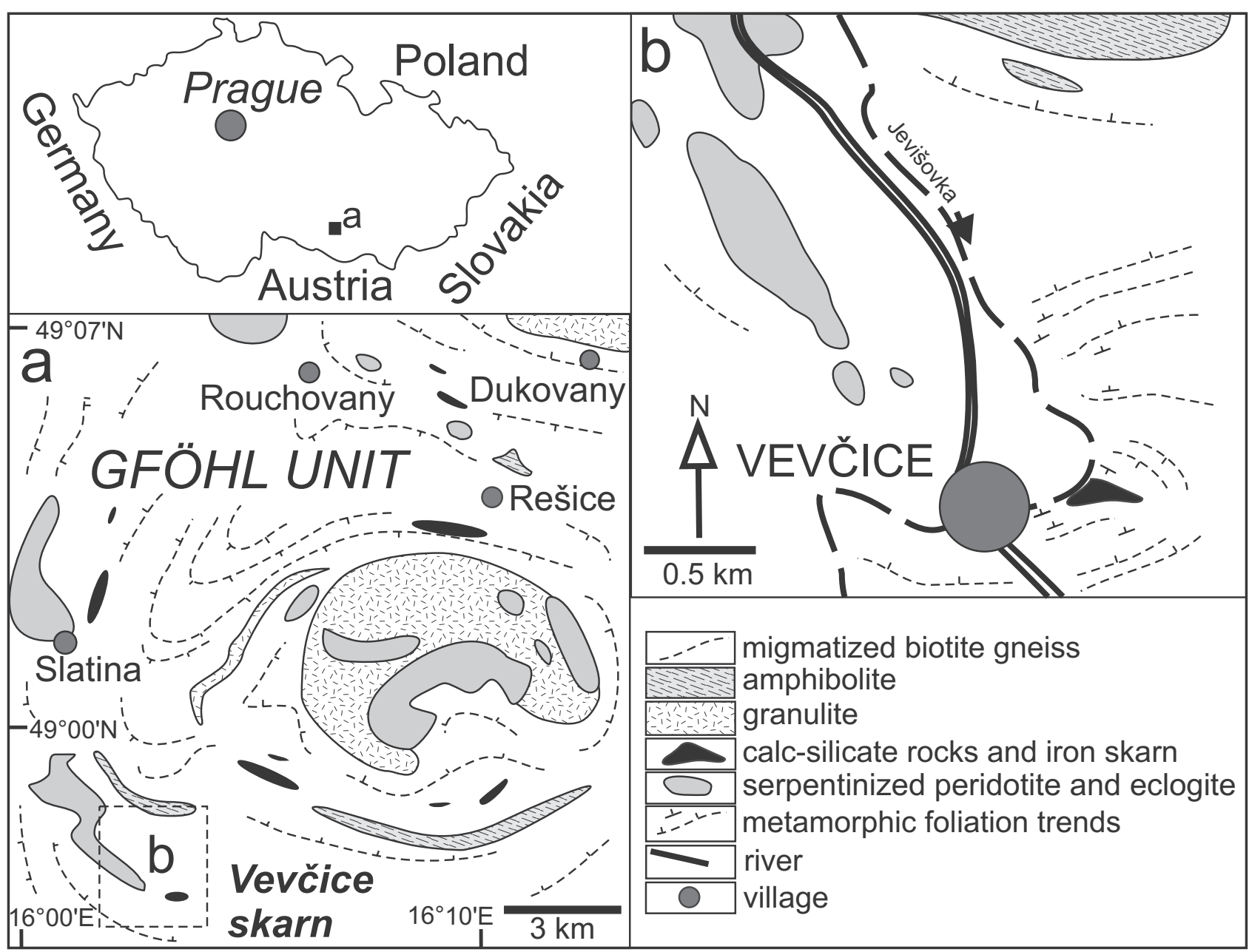

Fig. 1 Simplified geological map of the area of interest (modified from Homola et al. 1968; Martinec 1977; Matějovská et al. 1987).

were defined here (e.g., Fuchs and Matura 1976; Matte et al. 1990; Fiala et al. 1995; Schulmann et al. 2008):

(i) mid-crustal, amphibolite-facies Drosendorf Assemblage divided into the Monotonous (sillimanite-biotitecordierite migmatites with subordinate quartzites and amphibolites) and Varied units (migmatized paragneisses with numerous intercalations or small bodies of marbles, calc-silicate rocks and graphitic gneisses, locally also quartzites, amphibolites and orthogneisses);

(ii) lower-crustal/upper-mantle Gföhl Unit dominated by felsic orthogneisses to migmatites (the so-called Gföhl gneiss) and migmatized biotite paragneisses that are commonly associated with high-pressure felsic granulites. The granulites enclose serpentinized garnet/spinel peridotites, garnet pyroxenites, eclogites, iron-rich skarns, and in Raabs subunit also amphibolites, serpentinites, graphitic quartzites, and sporadic marbles and calc-silicate rocks (e.g. Fuchs and Matura 1976; Fritz 1995; Schulmann et al. 2005; Hasalová et al. 2008). The high-temperature (HT) and high-pressure (HP) peak metamorphic conditions of $800-900^{\circ} \mathrm{C}$ and $\sim 1.8 \mathrm{GPa}$ were documented in granulites and eclogites (Medaris et al. 1998; Štípská and
Powell 2005; Tajčmanová et al. 2006). The Gföhl gneiss is currently interpreted as felsic igneous rocks affected by metamorphism at upper amphibolite to granulite facies, more or less overprinted during a rapid decompression (Dudek et al. 1974; Cooke and O'Brien 2001). This is consistent with the data obtained for exhumation P-T path of the Gföhl gneiss (Hasalová et al. 2008), which indicate a decrease in equilibration temperature and pressure from $790^{\circ} \mathrm{C}$ and $0.6-0.9 \mathrm{GPa}$, to $690^{\circ} \mathrm{C}$ and $0.4-0.5$ GPa. The U-Pb zircon ages of 500-390 Ma obtained from the orthogneisses and granulites of the Gföhl Unit are interpreted as magmatic ages of their protolith (Friedl et al. 2004; Schulmann et al. 2005; Kusbach et al. 2015) whereas the ages ranging from 350 to $340 \mathrm{Ma}$ correspond to peak metamorphic conditions (e.g. Schulmann et al. 2005; Kusbach et al. 2015).

The studied assemblage (skarn penetrated by pegmatite) is part of a lithologically variable sequence of the Gföhl Unit in the area near Vevčice, north of Znojmo, Czech Republic (Fig. 1). This sequence is formed by strongly migmatized biotite gneisses which gradually change into nebulitic migmatites with small bodies of 
retrogressed granulites (biotite-sillimanite-garnet). Predominant biotite migmatites contain rare intercalations of skarn (e.g., Němec 1963b, 1991; Pertold et al. 1997; Pertoldová et al. 2009) and Ca-rich metasediments (Matějovská 1975; Matějovská et al. 1987; Houzar et al. 2014).

\subsection{Amphibole-rich pegmatite veins at skarn localities in the Bohemian Massif}

Amphibole-plagioclase pegmatite veins were found at several other localities in the Moldanubian Zone (including Kutná Hora and Svratka units at its N and NE boundaries; Němec 1991; Pertoldová et al. 2009). By contrast they are missing in the Saxothuringian Zone (Němec 1963a). The skarns associated with pegmatites occur at two tectono-stratigraphic levels in the Moldanubian Zone (Pertoldová et al. 2009, 2014): along the contact of the Gföhl Unit with the Varied Unit (Vlastějovice, Županovice), and within the Gföhl Unit (Rešice, Slatina, Vevčice and Kordula).

Mutual similarities among the pegmatites present at different skarn localities include in particular the mineral assemblage of amphibole + plagioclase \pm quartz, with minor K-feldspar, Fe-rich clinopyroxene, epidote, grossular-rich garnet and biotite, with accessory titanite and allanite (Němec 1963a, 1968, 1991). The pegmatite textures are usually comparable, with poorly evolved or absent zoning, and in some cases with biotite-amphibole reaction zones at contacts with (often pyroxene-rich) skarn host rocks.

Diorite pegmatites similar to those in the Vevčice skarn body occur elsewhere in the Gföhl Unit; the nearest localities are pyroxene and pyroxene-garnet skarns near Rešice and Slatina (Fig. 1a). They contain K-rich calcic amphiboles along with plagioclase $(\mathrm{Na}>\mathrm{Ca})$, locally clinopyroxene, K-feldspar and quartz, titanite, fluorapatite, and rare allanite and ferroaxinite (Filip et al. 2002). Analogous amphibole-rich pegmatite veins and the host skarn at Domanínek near Bystřice nad Pernštejnem are locally extremely enriched in allanite (Němec 1993).

The Vlastějovice skarn body is the only locality in the Bohemian Massif where pegmatites were studied in detail. Novák et al. (2013) identified four types of amphibole-rich pegmatite veins: (a) ordinary amphibolebearing pegmatites (resembling the Vevčice diorite pegmatites); (b) fluorite-rich pegmatites; (c) pegmatites with hydrothermal overprint and (d) pocket pegmatite. All four pegmatite types are characterized by amphibole, predominance of plagioclase over K-feldspar, quartz, biotite (annite) in variable amounts, locally abundant fluorite, allanite-(Ce) and REE-rich products of its hydrothermal alteration, calcite, (Al-F)-rich titanite, magnetite, rare epidote, clinopyroxene, apophyllite, prehnite, and sporadic tourmaline, ferroaxinite, wollastonite, uraninite, thorite and bismuth. Reaction rims composed of amphibole, biotite, and Ca-plagioclase (locally also fluorite) developed at the contact between (a) type amphibole pegmatites and the host skarn (Goliáš 2002; Žáček 2007; Novák et al. 2013).

\section{Analytical techniques}

\subsection{Mineral chemistry}

Electron microprobe analyses (EMPA) of individual minerals were performed using the Cameca SX-100 instrument at the Joint Laboratory of Electron Microscopy and Microanalysis of Masaryk University and the Czech Geological Survey (Brno, Czech Republic; analysts R. Škoda and P. Gadas). The measurements were carried out using a wavelength-dispersive (WDS) mode under the following conditions: accelerating voltage $15 \mathrm{kV}$, beam current 10 or $20 \mathrm{nA}$; beam diameter 1 or $4 \mu \mathrm{m}$. Natural and synthetic standards were used for amphibole, garnet, pyroxene, feldspars, epidote and accessory minerals: $\mathrm{Si}$, $\mathrm{Al}, \mathrm{K}$ - sanidine; $\mathrm{Na}$ - albite, $\mathrm{Fe}$ - almandine (andradite for feldspars), $\mathrm{Mn}$ - spessartine, $\mathrm{Mg}$ - pyrope, $\mathrm{Ca}$ - wollastonite, $\mathrm{Ti}$ - titanite, $\mathrm{P}$ - fluorapatite, $\mathrm{Y}-\mathrm{YPO}_{4}, \mathrm{Ni}$ - $\mathrm{Ni}_{2} \mathrm{SiO}_{4}, \mathrm{Zn}$ - gahnite, $\mathrm{Cr}$ - chromite, $\mathrm{Sc}, \mathrm{V}-\mathrm{ScVO}_{4}$, $\mathrm{Ba}$ - barite, $\mathrm{Sr}, \mathrm{S}-\mathrm{SrSO}_{4}, \mathrm{~F}$ - topaz, $\mathrm{Cl}$ - vanadinite, $\mathrm{REE}$ - phosphates of individual elements ( $\mathrm{La}, \mathrm{Ce}, \mathrm{Pr}, \mathrm{Nd}$, $\mathrm{Sm}, \mathrm{Gd}, \mathrm{Dy}, \mathrm{Er}$, and $\mathrm{Yb}$ ). The following standards were used for titanite, zircon, magnetite and ilmenite analyses: $\mathrm{Ti}$ - anatase, $\mathrm{Ca}, \mathrm{Si}$ - titanite, $\mathrm{Fe}$ - hematite, $\mathrm{Mg}$ - spinel, $\mathrm{Zr}$ - zircon, Hf - hafnium, U - metallic U, Th - brabantite, $\mathrm{Nb}$ - columbite, $\mathrm{Ta}-\mathrm{CrTa}_{2} \mathrm{O}_{6}$ and $\mathrm{Sn}$ - metallic $\mathrm{Sn}$. The raw data were processed using the $\mathrm{X}-\mathrm{Phi}$ correction of Merlet (1994).

The amphibole formulae were calculated on the basis of the cation sum of 13 atoms without $\mathrm{Ca}$, Na and K (13 eCNK) and the $\mathrm{Fe}^{2+}{ }_{\max } / \mathrm{Fe}^{3+}{ }_{\text {min }}$ ratios on the basis of 23 oxygen atoms (Leake et al. 1997). The abbreviations of the mineral names used in the text are from Whitney and Evans (2010).

\subsection{Whole-rock geochemistry}

Samples weighing $\sim 1 \mathrm{~kg}$ were used for whole-rock chemical analyses; they were acquired at the Acme Analytical Laboratories (Vancouver, Canada). Major oxides, $\mathrm{Ba}, \mathrm{Ni}, \mathrm{Cu}, \mathrm{Pb}$ and $\mathrm{Zn}$ were analysed by the ICP-ES method. Loss on ignition (LOI) was calculated from the weight difference after ignition at $1000^{\circ} \mathrm{C}$. The rare earth and other trace elements were analysed ICP-MS following $\mathrm{LiBO}_{2}$ fusion (analytic code: A4B4 - major oxides, Ba, Be, Co, Cr, Cs, Ga, Hf, Nb, Ni, Rb, Sc, Sr, Ta, Th, U, 
V, W, Y, Zr, REE; 1DX - Ag, As, Au, Bi, Cd, Cu, Hg, Mo, $\mathrm{Ni}, \mathrm{Pb}, \mathrm{Sb}, \mathrm{Se}, \mathrm{Tl}, \mathrm{Zn} ; 2$ ALeco $-\mathrm{C}_{\text {tot }}$, $\mathrm{S}_{\text {tot }}$; for analytical details, reproducibility, and detection limits see http:// acmelab.com). Geochemical data were processed using the GCDkit software package (Janoušek et al. 2006).

\subsection{Thermodynamic modelling}

The P-T histories of skarns are constrained through interpretation of reaction textures based on phase diagram (pseudosection) for model systems $\mathrm{CaO}-\mathrm{MgO}-\mathrm{FeO}-$ $\mathrm{Al}_{2} \mathrm{O}_{3}-\mathrm{SiO}_{2}-\mathrm{Na}_{2} \mathrm{O}-\mathrm{H}_{2} \mathrm{O}-\mathrm{CO}_{2}-\mathrm{O}_{2}$. The $\mathrm{P}-\mathrm{T}$ pseudosections for sample HS-2a have been calculated by the program PERPLEX (Connolly 2005) with the thermodynamic data of Holland and Powell (1998 updated in 2004). Mixing properties of phases used for the calculation were taken from Holland and Powell (1998): epidote and clinopyroxene, Massonne and Willner (2008): amphibole, Newton et al. (1980): plagioclase, White et al. (2007): garnet, Connolly and Cesare (1993): $\mathrm{C}-\mathrm{O}-\mathrm{H}$ fluid $(\mathrm{X}(\mathrm{O})=0.22 ; \mathrm{X}(\mathrm{O})=\mathrm{nO} /(\mathrm{nO}+\mathrm{nH})$, where $\mathrm{nO}$ and $\mathrm{nH}$ are the numbers of moles of oxygen and hydrogen, respectively).

\section{Results}

The skarn at Vevčice is a texturally variable body max. $\sim 30 \mathrm{~m}$ long; it is cross-cut by veins of diorite pegmatite up to $10 \mathrm{~cm}$ thick which form up to $25 \mathrm{vol}$. \% of the outcrop. The skarn is hosted by diatexite migmatite (Qz $+\mathrm{Kfs}+\mathrm{Pl}+\mathrm{Bt} \pm \mathrm{Grt} \pm \mathrm{Sil}$ ) which occasionally encloses layers and boudins of calc-silicate rocks (Pl + Di + Amp $+\mathrm{Ttn} \pm \mathrm{Rt}$ ) and dark garnet-bearing biotite gneiss (Houzar et al. 2014). Fine-grained leucocratic granulite (Qz + $\mathrm{Kfs}+\mathrm{Pl}+\mathrm{Grt}+\mathrm{Sil} \pm \mathrm{Ky} \pm \mathrm{Bt}$ ) was found in migmatites near the skarn footwall.

\subsection{Petrography}

\subsubsection{Skarn}

The skarn body is composed of three major rock types with distinct mineral assemblages:

(i) garnet + clinopyroxene \pm amphibole \pm plagioclase (skarn type A),

(ii) garnet + clinopyroxene + epidote \pm amphibole \pm plagioclase (skarn type B),

(iii) amphibole + clinopyroxene + quartz + magnetite \pm plagioclase \pm garnet (skarn type $\mathrm{C}$ ).

Skarn type A This prevailing rock type in the outcrops is medium-grained and has massive, granoblastic texture. The skarn consists of garnet (70-80 vol. \%), clinopyroxene (15-20 vol. \%), and variable amounts of amphibole ( $\leq 10$ vol. $\%)$ and plagioclase ( $\leq 10$ vol. $\%)$. Garnet is subhedral and intergrows with clinopyroxene; their textures indicate that they were early minerals in the skarn assemblage. Amphibole forms black subhedral to anhedral grains up to $15 \mathrm{~mm}$ across. It is younger than garnet and clinopyroxene, as it forms poikiloblasts with inclusions of these phases, or veinlets cross-cutting the garnet and pyroxene grains. Increased amounts of amphibole, typically subhedral to euhedral in shape, are present in fine-grained parts of the skarn. Subhedral plagioclase and rare anhedral quartz are irregularly distributed between garnets grains. Accessory minerals include magnetite (with exsolutions of aluminous spinel), locally intergrown with ilmenite, rare titanite and zircon.

Skarn type B This relatively rare skarn type is finegrained and granoblastic. Its mineral assemblage is dominated by garnet (50-70 vol. \%) with epidote (15-30 vol. \%) and small amounts of amphibole ( $<10$ vol. \%) and clinopyroxene ( $<10 \mathrm{vol} . \%)$. Texturally late epidote is commonly intergrown with garnet and locally forms fine-grained Grt + Ep + Pl symplectites. Accessory minerals include common titanite, minor plagioclase, and rare quartz. Late hydrothermal mineral assemblages (usually less than $5 \mathrm{~mm}$ thick veinlets) contain mainly epidoteclinozoisite, prehnite and albite.

Skarn type C The texturally heterogeneous skarn type $C$ is relatively rich in amphibole (40-70 vol. \%), plagioclase (10-40 vol. \%), clinopyroxene (10-30 vol. \%), magnetite (1-8 vol. \%), quartz (0-7 vol. \%) and plagioclase (0-3 vol. \%). Amphibole is younger than pyroxene and amphibole grains contain inclusions of garnet, K-feldspar, titanite, REE-epidote and allanite. Magnetite forms mm-sized intergrowths with pyroxene, amphibole and quartz. Accessory minerals are represented by fluorapatite, prevailing over zircon, ilmenite, and rutile.

\subsubsection{Diorite pegmatite}

The skarn body is brecciated and contains significant amounts of the amphibole-rich pegmatite matrix (10-50 vol. \% in the central part). The largest accumulations of pegmatite are observed near skarn footwall (Fig. 2a). The pegmatites are least abundant in the upper part of the skarn body where irregular narrow pegmatite veinlets form less than 10 vol. \% of the rock (Fig. 2b). The pegmatite veins are in all cases very irregular and their shape depends on degree and type of brittle deformation in the skarn body. The skarn xenoliths are typically rounded (Fig. 2a), and in some cases subangular and angular (Fig. 2c-d).

The pegmatite veins range in composition from tonalite to meladiorite (cumulatively designated as diorite pegmatite). Major pegmatite minerals are oligoclase-andesine plagioclase (40-63 vol. \%), amphibole (35-50 vol. \%) 

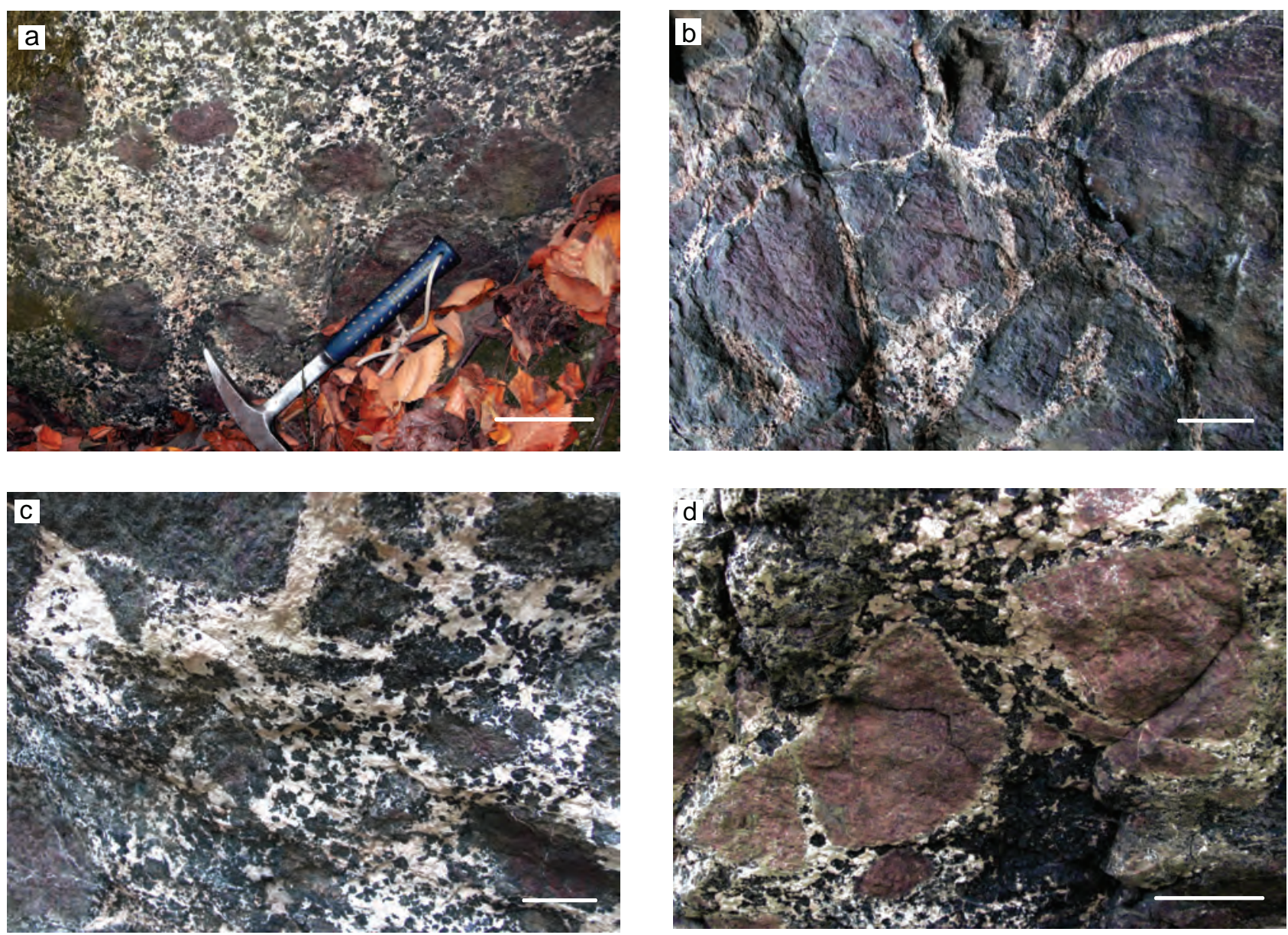

Fig. 2 Field relations of skarns and pegmatites: a - Rounded xenoliths of skarn (type A) in dominant diorite pegmatite; $\mathbf{b}$ - Diorite pegmatite veins, 1-3 cm thick, irregularly cutting type A skarn; c - Subangular to angular xenoliths of type C skarn in pegmatite, $\mathbf{d}$ - Angular xenoliths of type A skarn in pegmatite. All scale bars are $10 \mathrm{~cm}$ long.

and quartz (2-23 vol. \%); minor minerals are represented by K-feldspar (locally enclosed in plagioclase), garnet, allanite-epidote, clinopyroxene, and accessory titanite. Garnet typically forms small anhedral grains enclosed in amphibole, less frequently tiny inclusions in plagioclase. Quartz occurs as small anhedral grains among large subhedral plagioclase $\left(\mathrm{An}_{15-44}\right)$ and columnar amphibole crystals. The contacts between diorite pegmatite and skarn are typically sharp; narrow $(<5 \mathrm{~mm}$ ) plagioclase-rich reaction zones are locally present mainly adjacent to pyroxene-rich skarn fragments (Fig. 2d). On microscopic scale, are garnet crystals and aggregates frequently penetrated by veinlets with an assemblage of amphibole \pm plagioclase, rare quartz (< 5 vol. \%) and K-feldspar.

\subsection{Mineral chemistry}

Minerals that are the most significant for comparison between the skarn and the diorite pegmatite include amphibole, garnet, clinopyroxene, feldspars, and accessory titanite and metamict allanite. All (except sporadic clino- pyroxene in pegmatite) are present in variable amounts in both rock types.

\subsubsection{Amphibole}

Chemical composition of amphibole ranges from the K-rich hastingsite to potassic-hastingsite (Si 5.863-6.219 apfu, K 0.310-0.459 apfu, Na 0.291-0.504 apfu) with low contents of $\mathrm{Ti}(<0.212 \mathrm{apfu}), \mathrm{Cl}(<0.060 \mathrm{apfu})$ and Mn $(<0.034 \mathrm{apfu})$.

Hastingsite from diorite pegmatite is dominated by $\mathrm{K}$ over $\mathrm{Na}$ and low fraction of vacancies in the A-site $(0.250$ apfu). The amphibole has generally high $\mathrm{F}$ (>0.180 apfu), and also $\mathrm{Cl}$ (>0.035 apfu) when compared to the amphibole from skarn. Amphibole from the pegmatite at the contact with skarn has variable contents of $\mathrm{Ti}(>0.100$ apfu). Hastingsite crystals are insignificantly zoned, except for a slight increase in A-site vacancy, Fe, Si, and $\mathrm{F}$ in crystal rims in amphibole from pegmatite interior. This indicates substitutional trend towards ferro-edenite, or ferro-hornblende (Fig. 3a). 

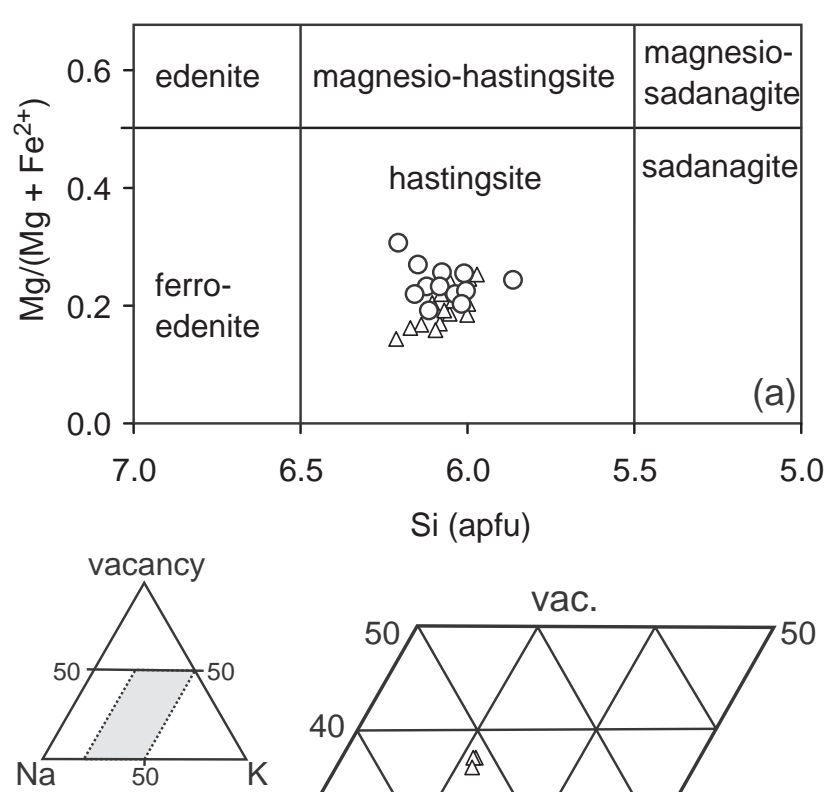

Si (apfu)

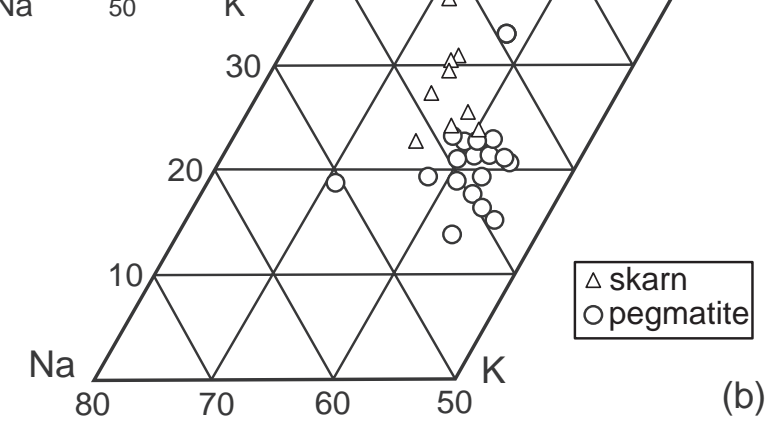

Fig. 3 Chemical composition of amphibole: $\mathbf{a}-$ Classification diagram $\mathrm{Mg} /\left(\mathrm{Mg}+\mathrm{Fe}^{2+}\right)$ vs. Si; $\mathbf{b}$ - Ternary diagram for the A-site occupancy.

Chemical composition of hastingsite from skarns shows weak variations in different mineral assemblages and textural settings (Tab. 1, Fig. 3b). Hastingsite from the skarn shows the highest proportion of vacancies in the A-site (0.22-0.38 apfu) and slight prevalence of $\mathrm{Na}$ over K. It has the lowest Ti (0.019-0.083 apfu) and $\mathrm{Cl}$ contents (0.008-0.031 apfu), particularly when associated with plagioclase.

\subsubsection{Garnet}

Assemblages with pyroxene in the skarn types $\mathrm{A}$ and $\mathrm{C}$ are dominated by Ca-rich almandine (Fig. 4; Tab. 2) with low contents of $\mathrm{Fe}^{3+}$ and very low $\mathrm{Mg}$ and $\mathrm{Mn}$ (Alm $\mathrm{Al}_{5-57}$ $\mathrm{Grs}_{30-37} \mathrm{Adr}_{3-9} \operatorname{Prp}_{5-7} \mathrm{Sps}_{1}$ ). Assemblages with epidote (skarn type B) are characterized by the presence of grossular-rich garnet $\left(\mathrm{Grs}_{45-53} \mathrm{Alm}_{20-29} \mathrm{Adr}_{16-34} \operatorname{Prp}_{1-4} \mathrm{Sps}_{1}\right)$. Fluorine was close to its detection limit (0.018-0.031 apfu) in all garnet types.

Garnet from the central parts of pegmatite (Fig. 4; Tab. 2) is Ca-rich almandine with slightly elevated amounts of spessartine component $\left(\mathrm{Alm}_{52-60} \mathrm{Grs}_{25-37} \mathrm{Adr}_{5-7}\right.$
$\left.\operatorname{Prp}_{3-7} \mathrm{Sps}_{1-3}\right)$. It has very low contents of Na (0.002-0.009 apfu), Ti (0.006-0.008 apfu), and F (0.005-0.018 apfu). In the vein border zones, garnet forms relict grains compositionally identical to the same mineral in the host skarn.

\subsubsection{Clinopyroxene}

Clinopyroxene is represented by Mg-rich hedenbergite (61-68 mol. \% Hd); clinopyroxene with elevated Fe contents is typical of assemblages with $\mathrm{Hd}+\mathrm{Amp}+\mathrm{Grt}$ \pm Mgt $\left(\mathrm{Fe}^{2+} 0.523-0.564\right.$ apfu in the garnet assemblages, and $\mathrm{Fe}^{2+} 0.538-0.613$ apfu in the magnetite assemblages, respectively; Tab. 3 ).

Compared to the other regionally metamorphosed skarns of the Gföhl Unit (Fig. 5), clinopyroxene from Vevčice has elevated contents of tetrahedral Al owing to the "Ca-Tschermak's component” substitution (Morimoto et al. 1988), particularly in the assemblages with garnet $\left({ }^{\mathrm{IV}} \mathrm{Al}=0.12-0.14 \mathrm{apfu}\right)$. The ${ }^{\mathrm{IV}} \mathrm{Al}$ contents do not reach the "fassaite" composition (defined as ${ }^{\mathrm{IV}} \mathrm{Al}>0.25 \mathrm{apfu}$ ) which was encountered in a skarn enclosed in the Blanský les granulite (Gföhl Unit) by Vrána (1987). Lower contents of ${ }^{\mathrm{IV}} \mathrm{Al}$ (0.052-0.094 apfu) were found in clinopyroxene from mineral assemblages with amphibole,
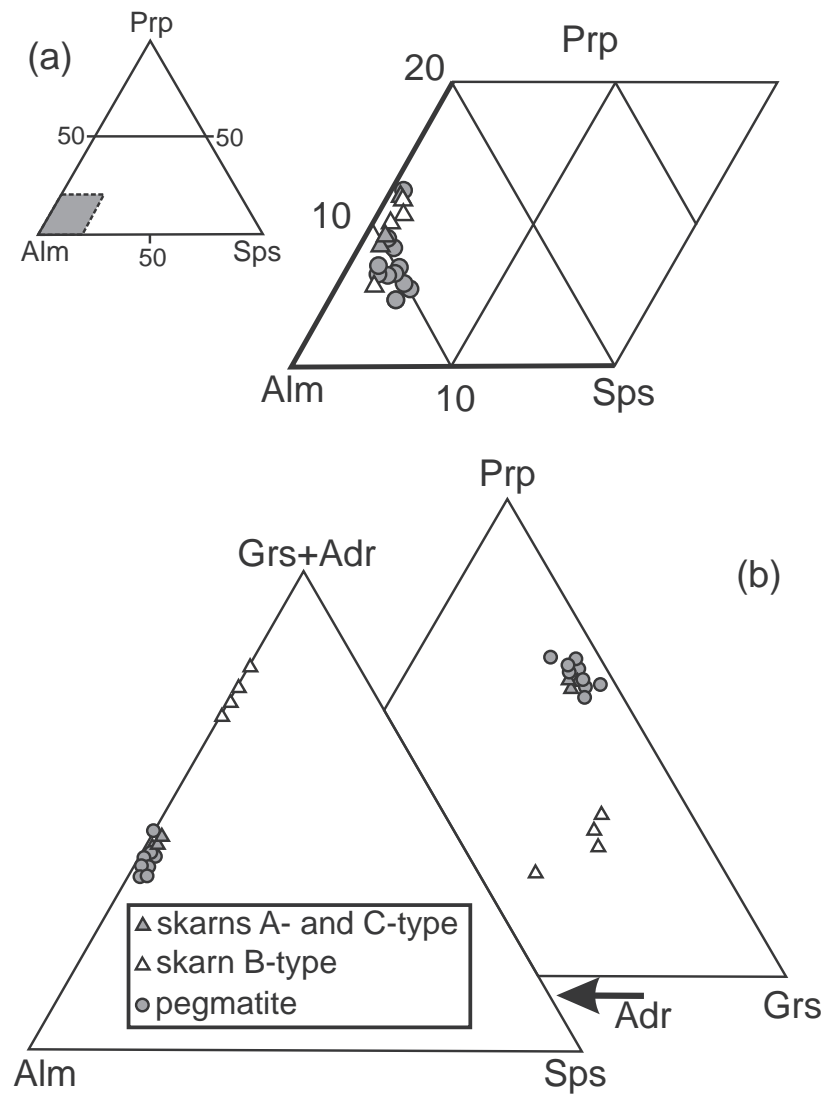

Fig. 4 Chemical composition of garnet from the skarns and diorite pegmatite veins: a - Alm-Prp-Sps; b -Alm-Grs + Adr-Sps, Adr-Prp-Grs (all mol. \%). 


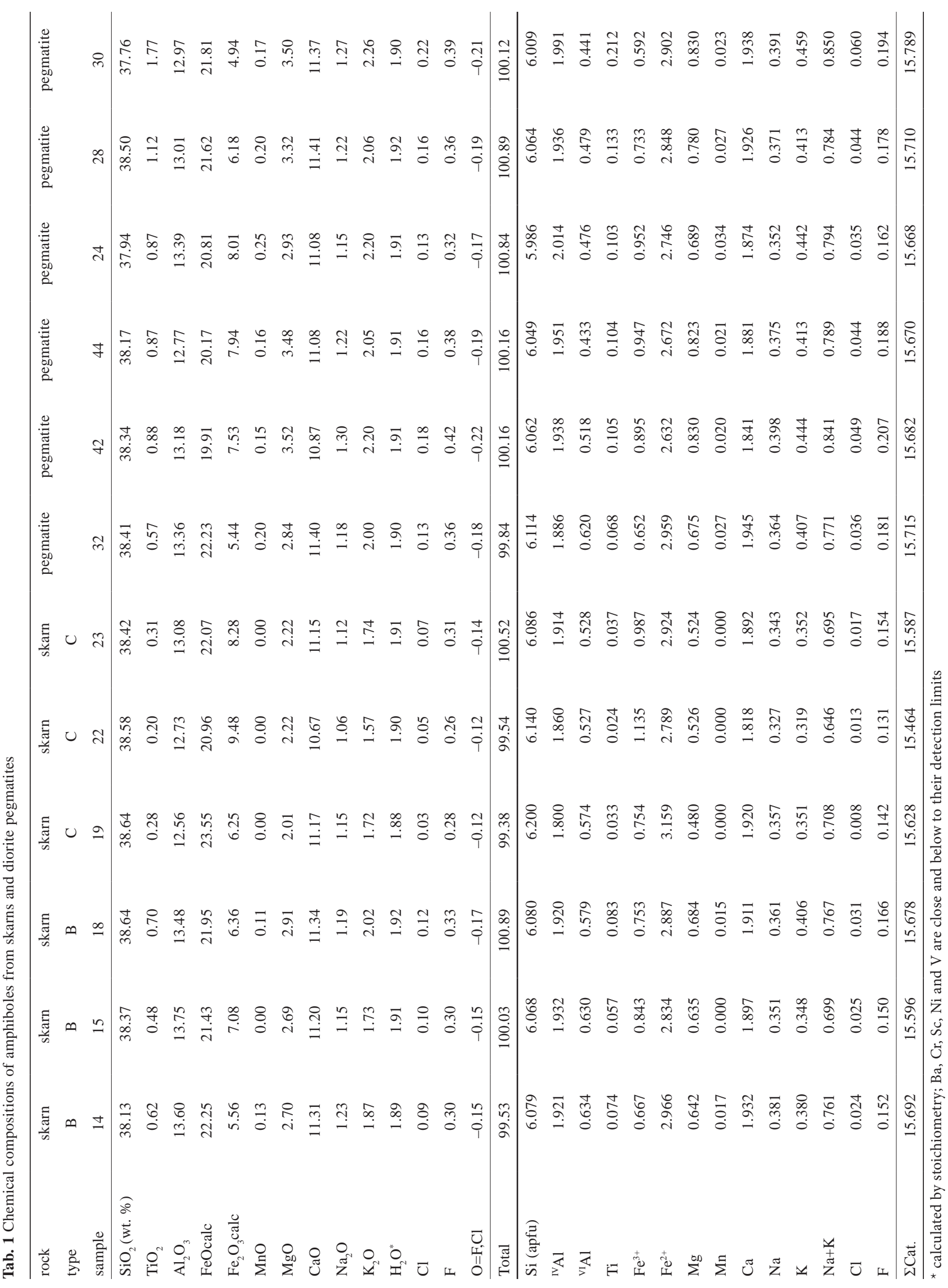


Tab. 2 Chemical compositions of garnets from skarns and diorite pegmatites

\begin{tabular}{|c|c|c|c|c|c|c|c|c|c|c|}
\hline rock & skarn & skarn & skarn & skarn & skarn & pegmatite & pegmatite & pegmatite & pegmatite & pegmatite \\
\hline type & A & A & B & B & C & & & & & \\
\hline sample & 51 & 3 & 18 & 21 & $29^{*}$ & 30 & 40 & 35 core & $36 \mathrm{rim}$ & $28 \mathrm{rim}$ \\
\hline $\mathrm{SiO}_{2}$ (wt. \%) & 37.99 & 38.12 & 37.99 & 38.20 & 37.96 & 37.13 & 37.33 & 37.58 & 36.99 & 37.37 \\
\hline $\mathrm{TiO}_{2}$ & 0.17 & 0.14 & 0.17 & 0.18 & 0.16 & 0.11 & 0.11 & 0.13 & 0.12 & 0.10 \\
\hline $\mathrm{Al}_{2} \mathrm{O}_{3}$ & 20.27 & 20.07 & 17.76 & 18.29 & 19.65 & 20.20 & 20.33 & 20.14 & 20.13 & 20.35 \\
\hline $\mathrm{Fe}_{2} \mathrm{O}_{3}$ & 2.24 & 2.86 & 6.41 & 5.48 & 2.68 & 2.01 & 2.00 & 1.91 & 1.71 & 1.59 \\
\hline $\mathrm{FeO}$ & 23.89 & 23.90 & 12.08 & 13.31 & 25.08 & 26.13 & 25.29 & 25.29 & 26.13 & 26.71 \\
\hline $\mathrm{MnO}$ & 0.39 & 0.23 & 0.22 & 0.20 & 0.34 & 0.93 & 0.91 & 0.53 & 1.18 & 1.31 \\
\hline $\mathrm{MgO}$ & 1.33 & 1.85 & 0.83 & 1.00 & 1.45 & 1.13 & 1.00 & 1.08 & 0.75 & 0.91 \\
\hline $\mathrm{CaO}$ & 14.66 & 14.19 & 24.78 & 23.84 & 13.68 & 12.08 & 13.09 & 13.48 & 12.28 & 11.92 \\
\hline Total & 100.97 & 101.39 & 100.25 & 100.50 & 101.00 & 99.78 & 100.10 & 100.23 & 99.33 & 100.30 \\
\hline Si (apfu) & 2.987 & 2.983 & 2.980 & 2.986 & 2.997 & 2.975 & 2.976 & 2.989 & 2.981 & 2.983 \\
\hline $\mathrm{Ti}$ & 0.010 & 0.008 & 0.010 & 0.011 & 0.009 & 0.007 & 0.007 & 0.008 & 0.007 & 0.006 \\
\hline $\mathrm{Al}$ & 1.878 & 1.851 & 1.642 & 1.685 & 1.828 & 1.908 & 1.910 & 1.888 & 1.912 & 1.915 \\
\hline $\mathrm{Fe}^{3+}$ & 0.133 & 0.169 & 0.378 & 0.322 & 0.159 & 0.121 & 0.120 & 0.115 & 0.104 & 0.096 \\
\hline $\mathrm{Fe}^{2+}$ & 1.571 & 1.565 & 0.793 & 0.870 & 1.656 & 1.751 & 1.686 & 1.682 & 1.761 & 1.783 \\
\hline $\mathrm{Mn}$ & 0.026 & 0.015 & 0.015 & 0.013 & 0.023 & 0.063 & 0.061 & 0.036 & 0.081 & 0.089 \\
\hline $\mathrm{Mg}$ & 0.156 & 0.216 & 0.097 & 0.117 & 0.171 & 0.135 & 0.119 & 0.128 & 0.090 & 0.108 \\
\hline $\mathrm{Ca}$ & 1.235 & 1.190 & 2.083 & 1.997 & 1.157 & 1.037 & 1.118 & 1.149 & 1.060 & 1.020 \\
\hline $\mathrm{O}$ & 12 & 12 & 12 & 12 & 12 & 12 & 12 & 12 & 12 & 12 \\
\hline Alm (mol. \%) & 52 & 52 & 26 & 29 & 55 & 58 & 56 & 56 & 59 & 59 \\
\hline Grs & 35 & 32 & 51 & 51 & 30 & 29 & 32 & 33 & 30 & 29 \\
\hline Adr & 7 & 9 & 19 & 16 & 8 & 6 & 6 & 6 & 5 & 5 \\
\hline Sps & 1 & 1 & 1 & 0 & 1 & 2 & 2 & 1 & 3 & 3 \\
\hline Prp & 5 & 7 & 3 & 4 & 6 & 5 & 4 & 4 & 3 & 4 \\
\hline
\end{tabular}

The formulae of garnet were obtained on the basis of 12 oxygen atoms and 8 cations

$\mathrm{Sc}$ a $\mathrm{Y}$ are below detecion limits; $\mathrm{Cr}, \mathrm{V}, \mathrm{Na}, \mathrm{P}$, and $\mathrm{F}$ close to their respective detection limits

* analysis taken from Houzar et al. (2014)

Tab. 3 Chemical compositions of clinopyroxene from skarns and diorite pegmatites

\begin{tabular}{|c|c|c|c|c|c|c|c|c|c|c|}
\hline rock & skarn & skarn & skarn & skarn & skarn & skarn & skarn & skarn & pegmatite & pegmatite \\
\hline type & A & A & A & A & B & B & C & $\mathrm{C}$ & & \\
\hline sample & 7 & $8^{*}$ & $27^{*}$ & 38 & $16^{*}$ & 12 & $50 *$ & 15 & 13 & 14 \\
\hline $\mathrm{SiO}_{2}$ (wt. \%) & 48.23 & 48.47 & 47.33 & 48.72 & 49.90 & 49.87 & 50.36 & 49.05 & 49.58 & 49.37 \\
\hline $\mathrm{TiO}_{2}$ & 0.39 & 0.35 & 0.30 & 0.24 & 0.19 & 0.15 & 0.09 & 0.27 & 0.19 & 0.24 \\
\hline $\mathrm{Al}_{2} \mathrm{O}_{3}$ & 3.72 & 3.65 & 4.00 & 3.52 & 2.34 & 1.93 & 1.01 & 3.48 & 2.30 & 2.81 \\
\hline $\mathrm{Fe}_{2} \mathrm{O}_{3}$ & 3.29 & 3.26 & 4.28 & 3.69 & 1.73 & 1.50 & 2.38 & 2.34 & 2.23 & 1.40 \\
\hline $\mathrm{FeO}$ & 16.13 & 16.33 & 17.23 & 16.85 & 17.07 & 17.53 & 18.92 & 16.62 & 17.3 & 17.58 \\
\hline $\mathrm{MnO}$ & 0.08 & 0.12 & 0.15 & 0.19 & 0.28 & 0.15 & 0.19 & 0.18 & 0.25 & 0.16 \\
\hline $\mathrm{MgO}$ & 6.64 & 6.68 & 5.65 & 5.90 & 6.76 & 6.66 & 6.15 & 6.72 & 6.56 & 6.32 \\
\hline $\mathrm{CaO}$ & 21.84 & 22.08 & 21.29 & 21.85 & 22.31 & 22.21 & 22.40 & 21.62 & 22.06 & 21.94 \\
\hline $\mathrm{Na}_{2} \mathrm{O}$ & 0.43 & 0.35 & 0.46 & 0.63 & 0.40 & 0.38 & 0.33 & 0.52 & 0.42 & 0.46 \\
\hline Total & 100.75 & 101.3 & 100.69 & 101.59 & 100.97 & 100.38 & 101.83 & 100.80 & 100.89 & 100.28 \\
\hline Si (apfu) & 1.872 & 1.872 & 1.853 & 1.883 & 1.931 & 1.944 & 1.952 & 1.898 & 1.925 & 1.925 \\
\hline $\mathrm{Ti}$ & 0.011 & 0.010 & 0.009 & 0.007 & 0.006 & 0.004 & 0.003 & 0.008 & 0.006 & 0.007 \\
\hline${ }^{\mathrm{IV}} \mathrm{Al}$ & 0.117 & 0.118 & 0.138 & 0.110 & 0.063 & 0.052 & 0.045 & 0.094 & 0.069 & 0.068 \\
\hline${ }^{\mathrm{VI}} \mathrm{Al}$ & 0.053 & 0.048 & 0.047 & 0.050 & 0.044 & 0.037 & - & 0.065 & 0.036 & 0.061 \\
\hline $\mathrm{Fe}^{3+}$ & 0.096 & 0.095 & 0.126 & 0.107 & 0.050 & 0.044 & 0.070 & 0.068 & 0.065 & 0.041 \\
\hline $\mathrm{Fe}^{2+}$ & 0.523 & 0.528 & 0.564 & 0.545 & 0.552 & 0.571 & 0.613 & 0.538 & 0.562 & 0.573 \\
\hline $\mathrm{Mn}$ & 0.003 & 0.004 & 0.005 & 0.006 & 0.009 & 0.005 & 0.006 & 0.006 & 0.008 & 0.005 \\
\hline $\mathrm{Mg}$ & 0.384 & 0.385 & 0.330 & 0.340 & 0.390 & 0.387 & 0.355 & 0.388 & 0.380 & 0.367 \\
\hline$\Sigma \mathrm{M} 1$ & 0.910 & 0.917 & 0.899 & 0.891 & 0.951 & 0.963 & 0.974 & 0.932 & 0.950 & 0.945 \\
\hline $\mathrm{Ca}$ & 0.908 & 0.914 & 0.893 & 0.905 & 0.925 & 0.927 & 0.930 & 0.896 & 0.918 & 0.917 \\
\hline $\mathrm{Na}$ & 0.032 & 0.026 & 0.035 & 0.047 & 0.030 & 0.029 & 0.025 & 0.039 & 0.032 & 0.035 \\
\hline$\Sigma$ M2 & 0.940 & 0.940 & 0.928 & 0.952 & 0.955 & 0.956 & 0.955 & 0.935 & 0.950 & 0.952 \\
\hline
\end{tabular}

Calculated on the basis of 6 anions and 4 cations

$\mathrm{Cr}, \mathrm{V}, \mathrm{Ni}, \mathrm{Zn}$ were below detection limits

* analyses taken from Houzar et al. (2014) 


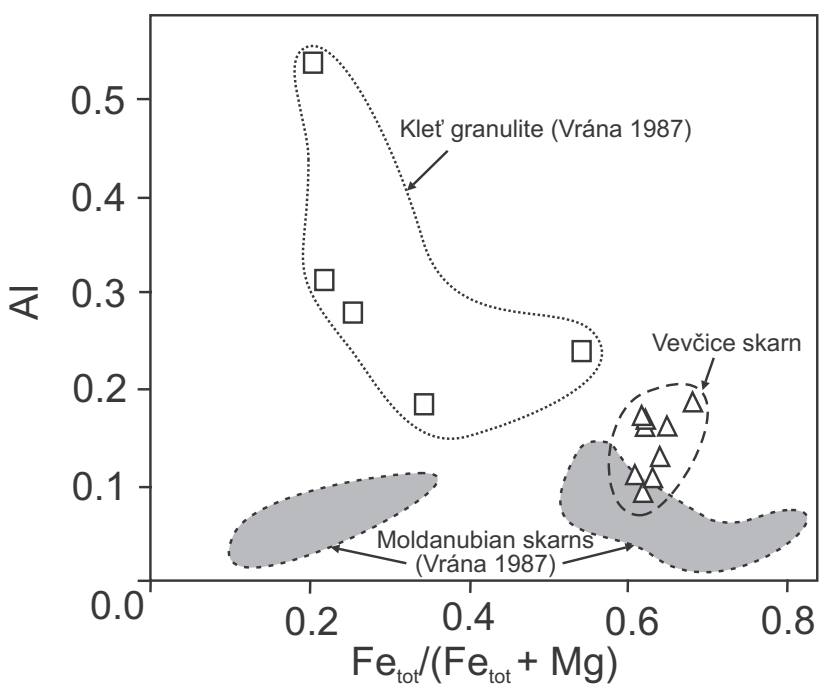

Fig. 5 Chemical composition of pyroxene illustrated by the $\mathrm{Al}$ vs. $\mathrm{Fe}_{\text {tot }}$ ' $\left(\mathrm{Fe}_{\mathrm{tot}}+\mathrm{Mg}\right)$ diagram (apfu).
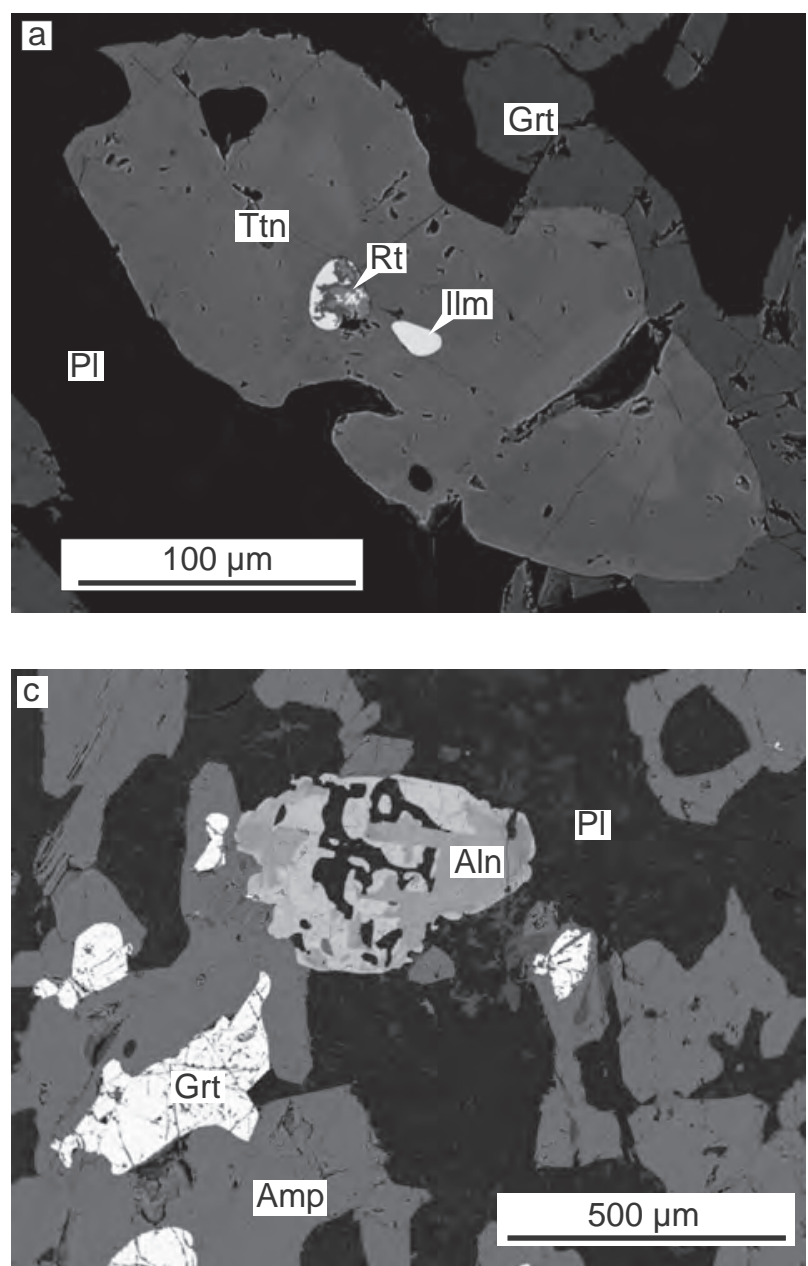

plagioclase, and quartz in skarn contact zones; and the lowest ( ${ }^{\mathrm{IV}} \mathrm{Al}<0.045 \mathrm{apfu}$ ) in assemblages with magnetite. Clinopyroxene has rather low contents of $\mathrm{Na}(0.025-$ $0.047 \mathrm{apfu})$ and $\mathrm{Fe}^{3+}(0.041-0.126 \mathrm{apfu})$, and is poor in Mn (0.003-0.009 apfu) and Ti (0.003-0.011 apfu).

\subsubsection{Feldspars}

Plagioclase is present in all rock types; it is mostly andesine $\left(\mathrm{An}_{28-46}\right)$ which typically occurs together with K-amphibole. Feldspars are volumetrically dominant especially in the diorite pegmatite which, in addition, contains oligoclase $\left(\mathrm{An}_{15-18}\right)$ with inclusions of K-feldspar (Houzar et al. 2014).

\subsubsection{Minor and accessory minerals}

Accessory titanite is present as microscopic grains in amphibole-bearing parts of both skarn and pegmatite
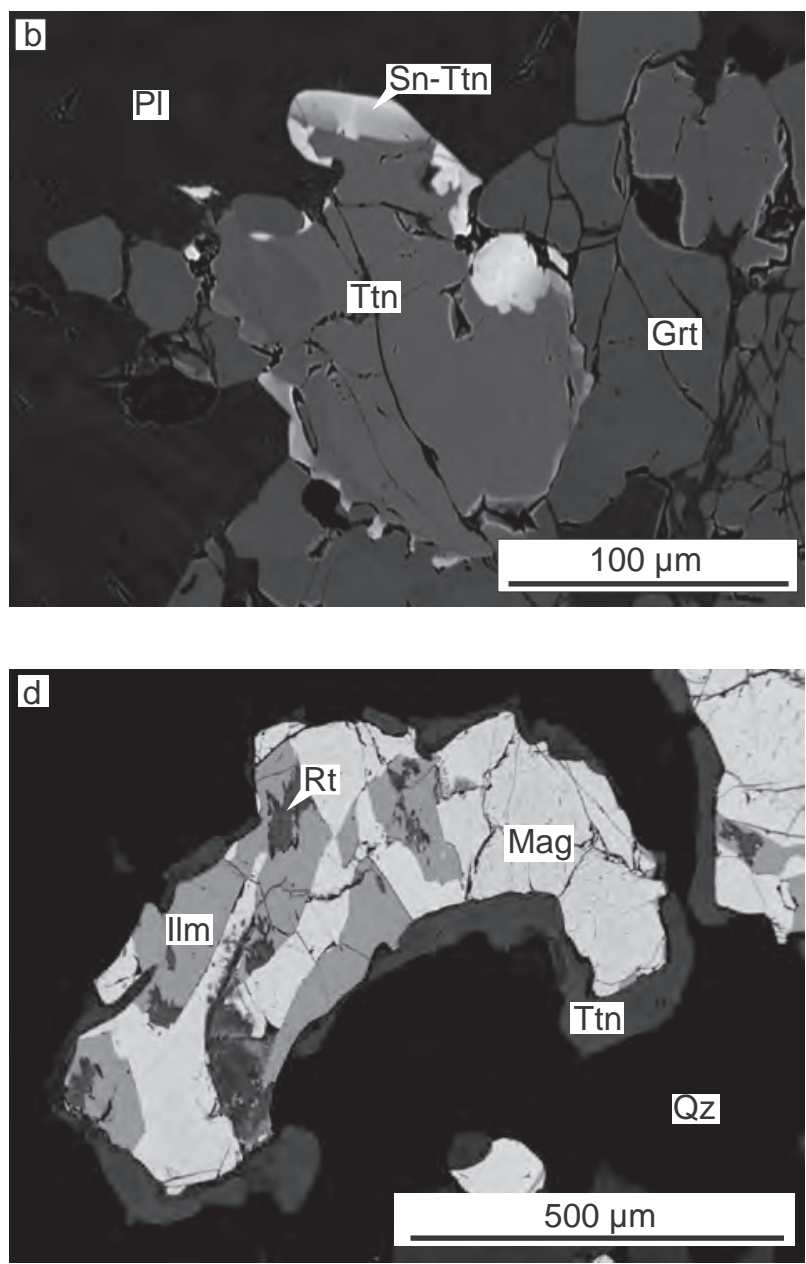

Fig. 6 Backscattered electron images of titanite- and allanite-bearing mineral assemblages and internal zoning. a - Patchy-zoned titanite with small inclusions of ilmenite \pm rutile surrounded by garnet and plagioclase; $\mathbf{b}$ - Titanite grain (pale grey) partially overgrown by Sn-rich titanite (white) in garnet (grey) and altered plagioclase (dark grey); c - Heterogeneous grain of allanite (pale grey, in the centre) associated with plagioclase (dark), amphibole (grey), and garnet (white) from a narrow, amphibole-rich veinlet in the skarn. $\mathbf{d}$ - Complex intergrowth of magnetite (bright, with exsolutions of Al-spinel and inclusions of rutile, top right) with ilmenite (darker grey), surrounded by a thin rim of titanite. All minerals are enclosed in quartz (black). 
Tab. 4 Representative chemical compositions of metamict allanite and titanite

\begin{tabular}{|c|c|c|c|c|c|c|c|c|c|}
\hline rock & skarn C & skarn C & pegmatit & skarn C & skarn A & skarn C & skarn B & skarn B & skarn B \\
\hline sample & 42 Aln & 43 Aln & 46 Aln & $47 \mathrm{Aln}^{*}$ & 59 Ttn & 33 Ttn & 1 Ttn & 6 Ttn & 25 Ttn \\
\hline $\mathrm{SiO}_{2}$ (wt. \%) & 37.28 & 37.87 & 42.76 & 37.86 & 30.91 & 30.64 & 30.34 & 30.05 & 28.95 \\
\hline $\mathrm{TiO}_{2}$ & 0.18 & 0.16 & 0.08 & 0.04 & 30.94 & 32.84 & 36.18 & 35.64 & 26.99 \\
\hline $\mathrm{ThO}_{2}$ & 1.10 & 1.06 & 1.47 & 0.56 & b.d. & b.d. & b.d. & b.d. & b.d. \\
\hline $\mathrm{SnO}_{2}$ & n.a. & n.a. & n.a. & n.a. & 0.96 & 1.22 & 1.36 & 3.71 & 11.89 \\
\hline $\mathrm{Al}_{2} \mathrm{O}_{3}$ & 17.00 & 16.85 & 14.72 & 19.74 & 3.31 & 2.48 & 2.76 & 2.24 & 1.99 \\
\hline $\mathrm{Fe}_{2} \mathrm{O}_{3}^{* *}$ & 9.18 & 9.57 & 8.02 & 10.28 & - & - & - & - & - \\
\hline $\mathrm{Ce}_{2} \mathrm{O}_{3}$ & 5.69 & 5.45 & 6.57 & 4.00 & 0.06 & 0.09 & 0.17 & 0.16 & b.d. \\
\hline $\mathrm{La}_{2} \mathrm{O}_{3}$ & 3.50 & 3.24 & 4.27 & 2.34 & b.d. & b.d. & b.d. & b.d. & b.d. \\
\hline $\mathrm{Nd}_{2} \mathrm{O}_{3}$ & 2.53 & 2.55 & 2.84 & 1.89 & 0.14 & 0.05 & 0.18 & 0.13 & 0.02 \\
\hline $\mathrm{Sm}_{2} \mathrm{O}_{3}$ & 0.23 & 0.30 & 0.27 & 0.12 & b.d. & b.d. & b.d. & b.d. & b.d. \\
\hline $\mathrm{Pr}_{2} \mathrm{O}_{3}$ & 0.74 & 0.79 & 0.97 & 0.53 & b.d. & b.d. & b.d. & b.d. & b.d. \\
\hline $\mathrm{Yb}_{2} \mathrm{O}_{3}$ & 0.04 & 0.06 & b.d. & 0.02 & b.d. & b.d. & b.d. & b.d. & b.d. \\
\hline $\mathrm{Nb}_{2} \mathrm{O}_{5}$ & n.a. & n.a. & n.a. & n.a. & 0.11 & 0.09 & 0.08 & 0.05 & 0.19 \\
\hline $\mathrm{FeO}^{*}$ & - & - & - & - & 1.36 & 1.55 & 1.02 & 1.15 & 1.03 \\
\hline $\mathrm{CaO}$ & 10.42 & 10.13 & 8.40 & 14.47 & 28.7 & 28.41 & 28.24 & 27.67 & 27.78 \\
\hline $\mathrm{MnO}$ & 0.19 & 0.20 & 0.39 & 0.26 & b.d. & b.d. & b.d. & b.d. & b.d. \\
\hline $\mathrm{SrO}$ & 0.08 & 0.04 & 0.02 & 0.25 & b.d. & b.d. & b.d. & b.d. & b.d. \\
\hline $\mathrm{Na}_{2} \mathrm{O}$ & n.a. & n.a. & n.a. & n.a. & b.d. & 0.03 & 0.09 & b.d. & 0.01 \\
\hline $\mathrm{F}$ & b.d. & b.d. & b.d. & b.d. & 1.18 & 0.91 & 0.94 & 0.86 & 0.78 \\
\hline $\mathrm{O}=\mathrm{F}$ & - & - & - & - & -0.50 & -0.38 & -0.40 & -0.36 & -0.33 \\
\hline $\mathrm{H}_{2} \mathrm{O}^{* * *}$ & 1.61 & 1.62 & 1.67 & 1.72 & - & - & - & - & - \\
\hline Total & 89.77 & 89.89 & 92.45 & 94.08 & 97.17 & 97.93 & 100.96 & 101.30 & 99.30 \\
\hline Si (apfu) & 3.476 & 3.510 & 3.848 & 3.303 & 1.025 & 1.014 & 0.981 & 0.983 & 0.997 \\
\hline $\mathrm{Ti}$ & 0.013 & 0.011 & 0.005 & 0.003 & 0.772 & 0.817 & 0.880 & 0.877 & 0.699 \\
\hline Th & 0.023 & 0.022 & 0.030 & 0.011 & - & - & - & - & - \\
\hline Sn & - & - & - & - & 0.013 & 0.016 & 0.018 & 0.048 & 0.163 \\
\hline $\mathrm{Al}$ & 1.868 & 1.841 & 1.561 & 2.030 & 0.129 & 0.097 & 0.105 & 0.086 & 0.081 \\
\hline $\mathrm{Fe}^{3+}$ & 0.644 & 0.667 & 0.543 & 0.675 & - & - & - & - & - \\
\hline $\mathrm{Ce}$ & 0.194 & 0.185 & 0.216 & 0.128 & 0.001 & 0.001 & 0.002 & 0.002 & \\
\hline $\mathrm{La}$ & 0.120 & 0.111 & 0.142 & 0.075 & - & - & - & - & - \\
\hline $\mathrm{Nd}$ & 0.084 & 0.084 & 0.091 & 0.059 & 0.002 & 0.001 & 0.002 & 0.002 & \\
\hline Sm & 0.007 & 0.010 & 0.008 & 0.004 & - & - & - & - & - \\
\hline $\operatorname{Pr}$ & 0.025 & 0.027 & 0.032 & 0.017 & - & - & - & - & - \\
\hline $\mathrm{Yb}$ & 0.001 & 0.002 & & 0.001 & - & - & - & - & - \\
\hline $\mathrm{Nb}$ & - & - & - & - & 0.002 & 0.001 & 0.001 & 0.001 & 0.003 \\
\hline $\mathrm{Fe}^{2+}$ & - & - & - & - & 0.038 & 0.043 & 0.028 & 0.031 & 0.030 \\
\hline $\mathrm{Ca}$ & 1.041 & 1.006 & 0.810 & 1.353 & 1.020 & 1.008 & 0.978 & 0.970 & 1.026 \\
\hline $\mathrm{Mn}$ & 0.015 & 0.016 & 0.030 & 0.019 & - & - & - & - & - \\
\hline $\mathrm{Sr}$ & 0.004 & 0.002 & 0.001 & 0.013 & - & - & - & - & - \\
\hline $\mathrm{Na}$ & - & - & - & - & - & 0.002 & 0.006 & & 0.001 \\
\hline $\mathrm{F}^{-}$ & - & - & - & - & 0.124 & 0.095 & 0.096 & 0.089 & 0.085 \\
\hline $\mathrm{H}^{+}$ & 1 & 1 & 1 & 1 & - & - & - & - & - \\
\hline$\Sigma$ Cat. & 7.516 & 7.493 & 7.319 & 7.689 & 3 & 3 & 3 & 3 & 3 \\
\hline $\mathrm{O}$ & 13 & 13 & 13 & 13 & 4.816 & 4.850 & 4.884 & 4.910 & 4.862 \\
\hline
\end{tabular}

The empirical formulae were normalized using $13 \mathrm{O}$ (epidote) or 3 cations (titanite), respectively.

n.a. not analyzed; b.d. below the detection limit

* analysis taken from Houzar et al. (2014)

** all $\mathrm{Fe}$ as $\mathrm{Fe}_{2} \mathrm{O}_{3}$ (allanite) or as $\mathrm{FeO}$ (titanite)

*** calculated by stoichiometry

(Fig. 6a). Its patchy-zoned grains are characterized by variable amounts of $\mathrm{Al}(0.081-0.151 \mathrm{apfu})$ and $\mathrm{F}$ (0.085-0.131 apfu) resulting from the substitution (Ti O)(Al F) ${ }_{-1}$ (e.g., Cempírek et al. 2008); the concentrations of Fe are rather low (0.019-0.046 apfu). Contents of $\mathrm{Nb}, \mathrm{Sn}, \mathrm{LREE}$, and $\mathrm{Na}$ are very low; however, rims of titanite grains are locally enriched in Sn $(>0.014$ apfu). The highest Sn amounts (0.048-0.163 apfu) were found in the titanite from the epidote-garnet skarn type B (Fig. 6b). 
Characteristic minor to accessory minerals in the pyroxeneamphibole skarn are REE-rich epidote and allanite, both typically metamict and hydrated (Houzar et al. 2014). These minerals are dominated by LREE (Ce 0.0750.216 apfu, La 0.059-0.142 apfu, Nd 0.059-0.091 apfu), whereas HREE $+\mathrm{Y}$ are below the detection limits (Tab. 4). Allanite is typically metamict, hydrated and Si-deficient; its alteration causes formation of (sub)microscopic chemically inhomogeneous grains with Th, Si, REE and P (rhabdophane, thorogummite) on fractures or in vicinity (Fig. 6c). Chemically variable younger clinozoisite-epidote (0.03-0.76 apfu Fe) replaces garnet in B type skarn (Houzar et al. 2014). Magnetite is present in accessory amount only (skarn type $\mathrm{A}$ and $\mathrm{C}$ ). Its contents of $\mathrm{Ti}$ and $\mathrm{Al}$ are elevated (Ti 0.022-0.087 apfu; $<1.48$ wt. \%, Al 0.044-0.066 apfu; Tab. 5). Mn-poor ilmenite (MnO <

$1.56 \mathrm{wt}$ \%) occurs intergrown with rutile as corroded relics in titanite. Intergrowths of ilmenite with magnetite, rutile and $\mathrm{Al}$-spinel inclusions are locally rimmed
Tab. 5 Representative chemical compositions of magnetite and ilmenite

\begin{tabular}{lccccc}
\hline $\begin{array}{l}\text { sample } \\
\text { type } \\
\text { mineral }\end{array}$ & $\begin{array}{c}\text { skarn } \\
\text { magnetite }\end{array}$ & $\begin{array}{c}\text { skarn } \\
\text { magnetite }\end{array}$ & $\begin{array}{c}\text { skarn } \\
\text { magnetite }\end{array}$ & $\begin{array}{c}\text { skarn } \\
\text { C } \\
\text { magnetite }\end{array}$ & $\begin{array}{c}\text { skarn } \\
\text { ilmenite }\end{array}$ \\
\hline $\mathrm{SiO}_{2}$ (wt. \%) & 0.06 & 0.09 & 0.09 & 0.09 & n.a. \\
$\mathrm{TiO}_{2}$ & 2.36 & 0.75 & 3.04 & 1.27 & 52.68 \\
$\mathrm{Al}_{2} \mathrm{O}_{3}$ & 1.48 & 0.97 & 1.33 & 1.36 & 0.04 \\
$\mathrm{~V}_{2} \mathrm{O}_{3}$ & 0.05 & 0.12 & 0.10 & 0.06 & n.a. \\
$\mathrm{Fe}_{2} \mathrm{O}_{3}$ & 62.54 & 66.26 & 61.13 & 65.02 & - \\
$\mathrm{FeO}_{\mathrm{MnO}}$ & 33.46 & 32.05 & 34.01 & 32.51 & $45.49 *$ \\
$\mathrm{MgO}$ & 0.06 & 0.04 & 0.07 & 0.07 & 1.56 \\
\hline $\mathrm{Total}^{4+}$ apfu) & b.d. & b.d. & 0.01 & 0.06 & b.d. \\
\hline $\mathrm{Ti}^{4+}$ & 100.09 & 100.40 & 99.88 & 100.55 & 99.95 \\
$\mathrm{Al}^{3+}$ & 0.002 & 0.003 & 0.003 & 0.003 & - \\
$\mathrm{V}^{3+}$ & 0.068 & 0.022 & 0.087 & 0.036 & 1.000 \\
$\mathrm{Fe}^{3+}$ & 0.066 & 0.044 & 0.060 & 0.061 & 0.001 \\
$\mathrm{Fe}^{2+}$ & 0.002 & 0.004 & 0.003 & 0.002 & - \\
$\mathrm{Mn}^{2+}$ & 1.792 & 1.901 & 1.755 & 1.857 & - \\
$\mathrm{Mg}^{2+}$ & 1.066 & 1.022 & 1.085 & 1.032 & 0.961 \\
\hline$\Sigma \mathrm{Cat}$. & 0.002 & 0.001 & 0.002 & 0.002 & 0.033 \\
$\mathrm{O}$ & - & - & 0.001 & 0.003 & - \\
\hline
\end{tabular}

magnetite calculated on the basis of $4 \mathrm{O}$ and 3 cations, ilmenite on $3 \mathrm{O}$

n.a. not analyzed; b.d. below the detection limit

* in ilmenite all Fe calculated as $\mathrm{FeO}$

$\mathrm{Mg}, \mathrm{Mn}$ and $\mathrm{Si}$ were close to, and $\mathrm{Ni}, \mathrm{Cr}$, Sn with Zn already below, the respective detection limits

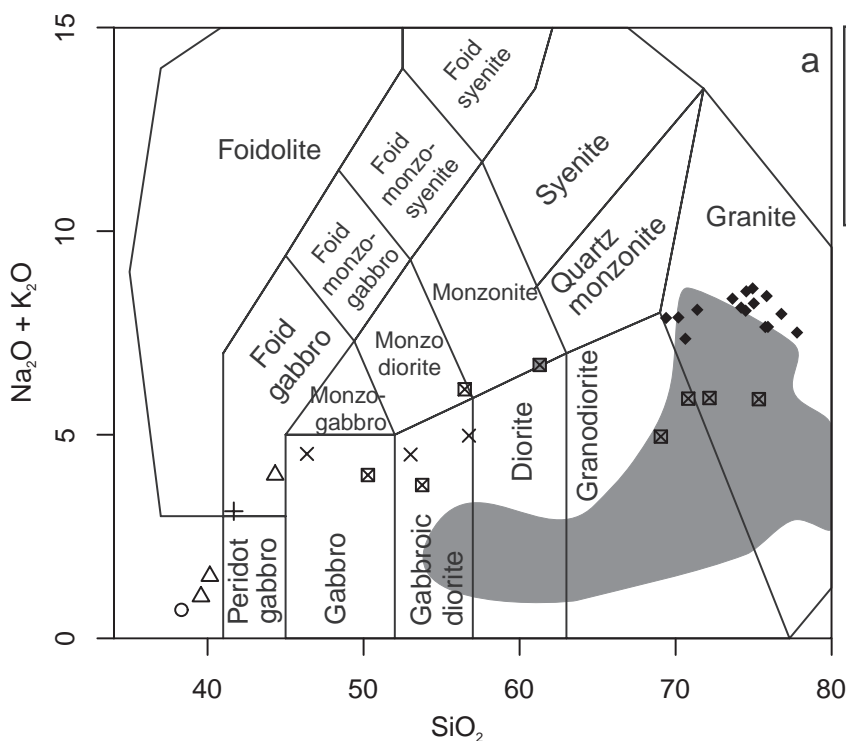

by titanite crystals (Fig. 6d). The rare accessories are fluorapatite $(\leq 2.28$ wt. $\% \mathrm{~F} ;<1$ wt. $\% \mathrm{Cl})$ and low- $\mathrm{Hf}$ zircon $\left(1.5-1.6\right.$ wt. \% $\left.\mathrm{HfO}_{2}\right)$.

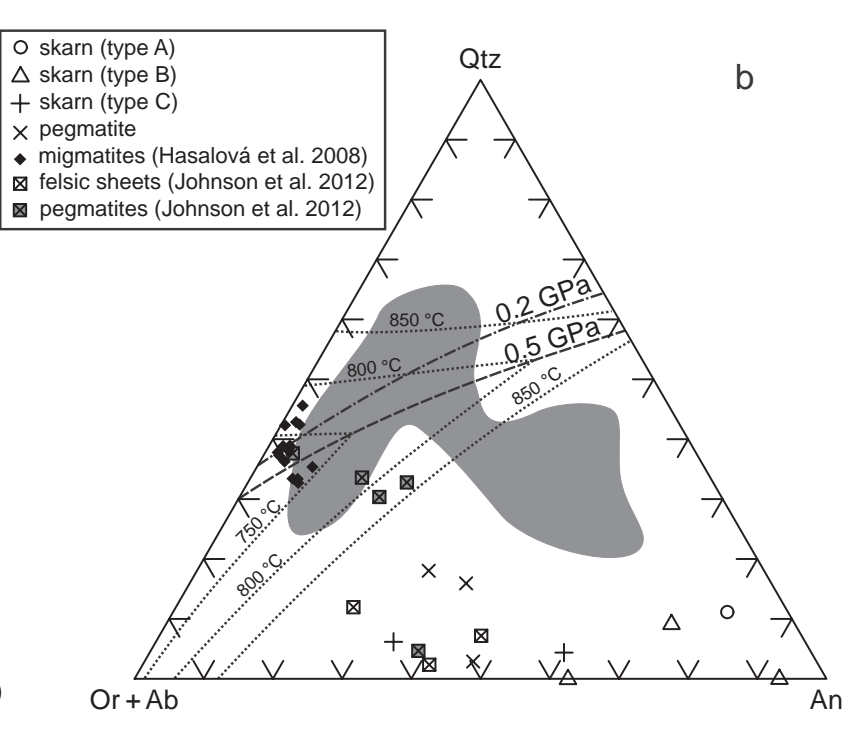

Fig. 7 Whole-rock chemical compositions of the skarns, diorite pegmatites (both this study), and migmatites from the Gföhl Unit (Hasalová et al. 2008). For comparison are shown chemistries of felsic sheets and pegmatites which are interpreted as product of amphibole dehydration melting and subsequent fractional crystallization (Johnson et al. 2012): a - Total alkali versus silica (TAS) diagram (Middlemost 1994), b - Normative $\mathrm{Ab}(+\mathrm{Or})-\mathrm{Qtz}-\mathrm{An}$ plot (based on the Improved Granite Mesonorm of Mielke and Winkler 1979). Liquidus phase relations at $0.5 \mathrm{GPa}$ and cotectic curve at $2 \mathrm{GPa}$ in the system Qtz- $\mathrm{Ab}-\mathrm{An}-\mathrm{H}_{2} \mathrm{O}$ are taken from Yoder (1968). The grey field denotes the liquid compositions from selected amphibole dehydration-melting experiments (Hacker 1990; Skjerlie and Johnston 1992; Wolf and Wyllie 1994; Watkins et al. 2007). 
Tab. 6 Major-element (wt. \%) whole-rock chemical compositions for individual skarn types and pegmatite

\begin{tabular}{|c|c|c|c|c|c|c|c|c|}
\hline $\begin{array}{l}\text { sample } \\
\text { rock } \\
\text { type }\end{array}$ & $\begin{array}{c}\text { HS-1 } \\
\text { skarn } \\
\text { A }\end{array}$ & $\begin{array}{c}\text { HS-3 } \\
\text { skarn } \\
\text { B }\end{array}$ & $\begin{array}{c}\text { HS-2 } \\
\text { skarn } \\
\text { B }\end{array}$ & $\begin{array}{c}\text { HS-2a } \\
\text { skarn } \\
\text { B }\end{array}$ & $\begin{array}{c}\text { HS-5 } \\
\text { skarn } \\
\text { C }\end{array}$ & $\begin{array}{c}\text { HS-4 } \\
\text { pegmatite }\end{array}$ & $\begin{array}{c}\text { HS-4a } \\
\text { pegmatite }\end{array}$ & $\begin{array}{c}\text { HS-4ag } \\
\text { pegmatite }\end{array}$ \\
\hline $\mathrm{SiO}_{2}$ & 38.93 & 40.49 & 42.97 & 38.97 & 41.66 & 46.17 & 56.10 & 52.62 \\
\hline $\mathrm{TiO}_{2}$ & 0.39 & 0.57 & 0.64 & 0.53 & 0.57 & 0.38 & 0.31 & 0.34 \\
\hline $\mathrm{Al}_{2} \mathrm{O}_{3}$ & 19.25 & 20.17 & 21.80 & 22.98 & 18.66 & 19.40 & 17.11 & 17.80 \\
\hline $\mathrm{Fe}_{2} \mathrm{O}_{3(\mathrm{tot})}$ & 26.25 & 23.28 & 12.53 & 13.70 & 22.28 & 17.49 & 11.22 & 14.23 \\
\hline $\mathrm{MnO}$ & 0.21 & 0.17 & 0.09 & 0.11 & 0.20 & 0.15 & 0.09 & 0.12 \\
\hline $\mathrm{Cr}_{2} \mathrm{O}_{3}$ & 0.00 & 0.01 & 0.01 & 0.01 & 0.01 & 0.00 & 0.00 & 0.00 \\
\hline $\mathrm{MgO}$ & 2.24 & 1.29 & 0.64 & 0.20 & 1.94 & 1.62 & 1.47 & 1.42 \\
\hline $\mathrm{CaO}$ & 13.47 & 13.17 & 14.00 & 21.42 & 11.33 & 10.80 & 7.53 & 8.12 \\
\hline $\mathrm{Na}_{2} \mathrm{O}$ & 0.46 & 1.70 & 2.16 & 0.65 & 1.84 & 3.28 & 3.59 & 2.93 \\
\hline $\mathrm{K}_{2} \mathrm{O}$ & 0.25 & 0.47 & 1.73 & 0.36 & 1.28 & 1.23 & 1.33 & 1.55 \\
\hline $\mathrm{P}_{2} \mathrm{O}_{5}$ & 0.13 & 0.18 & 0.37 & 0.18 & 0.19 & 0.13 & 0.10 & 0.13 \\
\hline LOI & 0.00 & 0.00 & 2.80 & 1.20 & 0.00 & 0.30 & 1.00 & 0.60 \\
\hline Total & 101.58 & 101.50 & 99.74 & 100.31 & 99.96 & 100.95 & 99.85 & 99.86 \\
\hline
\end{tabular}

Sample descriptions: HS-1 massive pyroxene-garnet skarn, HS-2 pyroxene-garnet-epidote skarn (fine-grained) with narrow amphibole + plagioclase veinlets, HS-2a pyroxene-garnet-epidote skarn (medium-grained), HS-3 garnet skarn (medium-grained), with rare narrow amphibole veinlets, HS-5 magnetite-bearing, amphibole-pyroxene \pm garnet \pm quartz skarn, HS-4 pegmatite with rare small amphibole-rich skarn relics, HS-4a diorite pegmatite, HS-4ag garnet-bearing amphibole pegmatite

\section{Whole-rock geochemistry}

\subsection{Skarns}

The whole-rock chemical composition was determined for representative samples of the three main skarn types (Tab. 6): (a) most common type A (HS-1), (b) minor skarn type B (HS-2, 2a, 3) and (c) sporadic skarn type C (HS-5).

Skarns are ultrabasic rocks (Fig. 7a, Tab. 6) with low contents of $\mathrm{Na}_{2} \mathrm{O}\left(0.46-2.16\right.$ wt. \%) and $\mathrm{K}_{2} \mathrm{O}(0.25-1.73$ wt. \%). Contents of major-element oxides and trace elements exhibit relatively large scatter (Figs 7b, 8, and Tab. 6). Garnet-pyroxene-amphibole skarn (type A) is characterized by low concentrations of $\mathrm{TiO}_{2}, \mathrm{Na}_{2} \mathrm{O}$, $\mathrm{P}_{2} \mathrm{O}_{5}$, LILE (large-ion lithophile elements), $\mathrm{Y}, \mathrm{Th}, \mathrm{U}, \mathrm{Zr}$ and $\mathrm{Nb}$ (Fig. 8, Tab. 6) as well as high contents of $\mathrm{FeO}_{\mathrm{t}}$ (23.6 wt. \%) and $\mathrm{MgO}$ (2.2 wt. \%). Epidote-rich (type B) skarns show lower contents of $\mathrm{MgO}(0.2-1.3 \mathrm{wt}$ \% \%) and high contents of $\mathrm{Al}_{2} \mathrm{O}_{3}, \mathrm{CaO}, \mathrm{Sr}, \mathrm{Y}, \mathrm{Th}, \mathrm{U}, \mathrm{Nb}$ and LREE (Figs 8, 9a) in comparison to the other skarn types. Skarn type $\mathrm{C}$ is characterized by relatively low contents of $\mathrm{Al}_{2} \mathrm{O}_{3}$ and $\mathrm{CaO}$ (Fig. 8).

The total REE contents are variable, ranging from 72 ppm to $298 \mathrm{ppm}$, the highest concentration being found in the epidote-rich skarn type B. Chondrite-normalized REE patterns (Boynton 1984) of the skarn type B are enriched in LREE $\left(\mathrm{La}_{\mathrm{N}} / \mathrm{Yb}_{\mathrm{N}}=5.2-8.1\right)$; on the other hand, garnetrich skarn type $\mathrm{A}$ is slightly depleted in $\operatorname{LREE}\left(\mathrm{La}_{\mathrm{N}} / \mathrm{Yb}_{\mathrm{N}}=\right.$ $0.9)$. The skarn type $C$ shows relatively flat REE pattern $\left(\mathrm{La}_{\mathrm{N}} / \mathrm{Yb}_{\mathrm{N}}=1.5\right)$. In general, the LREE-rich samples are characterized by the presence of allanite whereas LREE depletion correlates with a high proportion of garnet in the rock. All REE patterns share distinct positive Eu anomaly $\left(\mathrm{Eu} / \mathrm{Eu}^{*}=1.8-4.0\right)$ combined with relatively flat HREE segment (Fig. 9a).

Primitive mantle-normalized patterns (after McDonough and Sun 1995) for skarns (Fig. 9b) show considerable degree of enrichment in incompatible elements. All patterns are characterized by the presence of Th, U, Eu spikes and $\mathrm{Ba}, \mathrm{Nb}, \mathrm{Sr}$ and Ti troughs. The skarn type $\mathrm{B}$ is characterised by variable $\mathrm{Pb}, \mathrm{Sr}$ contents which correlate positively with modal amount of epidote. The garnet-rich skarn type A shows low contents of LILE, REE and HFSE (high field strength elements, mainly $\mathrm{Nb}$, Ta) in comparison with the type B.

\subsection{Diorite pegmatite}

The diorite pegmatite samples (Fig. 8, Tabs 6-7) are characterized by low contents of $\mathrm{SiO}_{2}(46.2-56.1$ wt. \%), $\mathrm{K}_{2} \mathrm{O}$ (1.2-1.6 wt. \%) and $\mathrm{Rb}(43-86 \mathrm{ppm})$, as well as high contents of $\mathrm{FeO}_{\mathrm{t}}(10.1-15.7$ wt. \%), $\mathrm{CaO}$ (7.5-10.1 wt. \%), $\mathrm{MgO}$ (1.4-1.6 wt. \%), and $\mathrm{Na}_{2} \mathrm{O}$ (2.9-3.3 wt. \%). In the TAS (total alkalis versus silica) diagram (Middlemost 1994), the pegmatite samples have a gabbroic to gabbrodioritic composition $\left(\mathrm{K}_{2} \mathrm{O}+\right.$ $\mathrm{Na}_{2} \mathrm{O}=4.5-5.0$ wt. \%, Fig. 7a). Variations in contents of main oxides correlate with the modal heterogeneity of the rock, mainly with the variation of amphibole/ plagioclase ratio.

The diorite pegmatites have variable, often low contents of REE (Tab. 7, 83-346 ppm), with a slight LREE/ HREE fractionation $\left(\mathrm{La}_{\mathrm{N}} / \mathrm{Yb}_{\mathrm{N}}=2.0-12.9\right.$; using normalization values from Boynton 1984), positive Eu anomaly $\left(\mathrm{Eu} / \mathrm{Eu}^{*}=1.3-1.6\right)$. The primitive mantle-normalized 

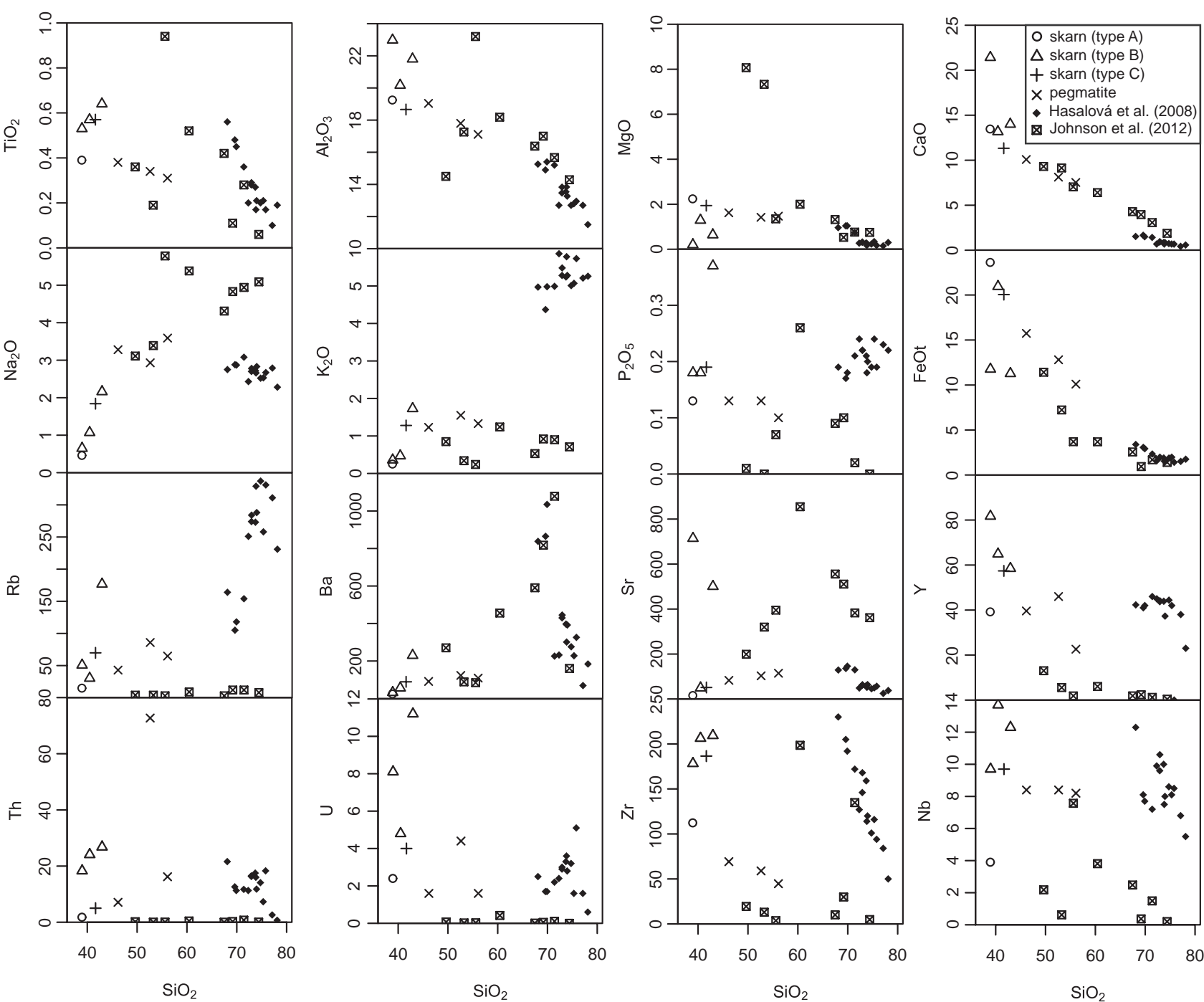

Fig. 8 Variation diagrams showing selected major-element oxide and trace-element abundances in individual types of skarn and in diorite pegmatite (both this study), and migmatites from the Gföhl Unit (Hasalová et al. 2008). For comparison are shown chemistries of felsic sheets and pegmatites that are interpreted as products of amphibole dehydration melting and subsequent fractional crystallization (Johnson et al. 2012).

patterns (after McDonough and Sun 1995) for pegmatites are similar to skarns (Fig. 9b), with variable concentrations of Th (7.1-72.7 ppm), Pb (2.9-5.9 ppm) and $\mathrm{Sr}$ (83.1-114.7 ppm). The abundances of $\mathrm{Rb}, \mathrm{Pb}$ and Eu are lower relative to the migmatites in Gföhl Unit (Figs 8, 9b; Hasalová et al. 2008).

\section{Discussion}

\subsection{Amphibole and garnet composition}

Amphiboles of the Vevčice skarn and diorite pegmatite have very similar compositions ranging from potassichastingsite to hastingsite (simple homovalent substitution $\mathrm{KNa}_{-1}$ ) and they are relatively poor in $\mathrm{Cl}$ and $\mathrm{F}$.
Amphiboles in pegmatite have a slightly lower A-site vacancy than those in skarns, and typically show slight prevalence of $\mathrm{K}$ over $\mathrm{Na}$ in the A-site. Slight difference is visible between compositional trends in hastingsite from the skarn and the pegmatite, whereby the latter, associated with plagioclase, is slightly enriched in Si and Mg.

Potassium-dominant amphiboles originate by three principal geological processes (Gulyaeva et al. 1986; Suwa et al. 1987; Mazdab 2003; Krmíček et al. 2011): (1) alkali-chloride metasomatism, (2) K-rich in-situ isochemical metamorphism, and (3) crystallization from K-rich magma and by affiliated metasomatism. But amphiboles in the present study do not belong to any of the groups above.

Potassium-rich amphiboles are known from several skarn bodies in the Moldanubian Zone (e.g. Holšice; 
Tab. 7 Trace-element (ppm) whole-rock chemical compositions for individual skarn types and pegmatite

\begin{tabular}{|c|c|c|c|c|c|c|c|c|}
\hline $\begin{array}{l}\text { sample } \\
\text { rock } \\
\text { type }\end{array}$ & $\begin{array}{c}\text { HS-1 } \\
\text { skarn } \\
\text { A }\end{array}$ & $\begin{array}{c}\text { HS-3 } \\
\text { skarn } \\
\text { B }\end{array}$ & $\begin{array}{c}\text { HS-2 } \\
\text { skarn } \\
\text { B }\end{array}$ & $\begin{array}{c}\text { HS-2a } \\
\text { skarn } \\
\text { B }\end{array}$ & $\begin{array}{c}\text { HS-5 } \\
\text { skarn } \\
\text { C }\end{array}$ & $\begin{array}{c}\text { HS-4 } \\
\text { pegmatite }\end{array}$ & $\begin{array}{c}\text { HS-4a } \\
\text { pegmatite }\end{array}$ & $\begin{array}{c}\text { HS-4ag } \\
\text { pegmatite }\end{array}$ \\
\hline As & 2.6 & 4.0 & 8.5 & 8.1 & 4.6 & 3.7 & 5.3 & 6.3 \\
\hline $\mathrm{Ba}$ & 24 & 56 & 230 & 32 & 89 & 91 & 109 & 123 \\
\hline $\mathrm{Be}$ & 2 & 2 & 3 & 3 & 3 & 6 & 12 & 13 \\
\hline $\mathrm{Bi}$ & 0.5 & 1.0 & 10.8 & 11.7 & 0.7 & 0.4 & 0.4 & 0.7 \\
\hline Co & 26.4 & 19.3 & 5.0 & 2.4 & 21.2 & 14.8 & 12.4 & 16.5 \\
\hline Cs & 0.5 & 0.8 & 6.0 & 1.2 & 1.5 & 1.1 & 2.0 & 2.4 \\
\hline $\mathrm{Cu}$ & 1.2 & 0.8 & 1.1 & 1.2 & 1.3 & 1.0 & 2.0 & 1.9 \\
\hline $\mathrm{Ga}$ & 34.5 & 37.2 & 56.5 & 74.7 & 34.0 & 34.8 & 33.5 & 33.1 \\
\hline $\mathrm{Hf}$ & 3.0 & 6.5 & 6.8 & 4.6 & 5.3 & 2.6 & 1.4 & 1.9 \\
\hline Mo & 0.1 & 0.1 & 0.2 & $<0.1$ & 0.2 & 0.1 & 0.1 & 0.2 \\
\hline $\mathrm{Nb}$ & 3.9 & 13.7 & 12.3 & 9.7 & 9.7 & 8.4 & 8.2 & 8.4 \\
\hline $\mathrm{Ni}$ & 7.1 & 5.3 & 5.3 & 1.2 & 8.2 & 6.8 & 12.5 & 10.8 \\
\hline $\mathrm{Pb}$ & 0.5 & 2.1 & 6.7 & 3.6 & 1.7 & 2.9 & 4.3 & 5.9 \\
\hline $\mathrm{Rb}$ & 14.9 & 30.3 & 176.7 & 50.7 & 69.8 & 43.0 & 64.8 & 85.9 \\
\hline $\mathrm{Sb}$ & $<0.1$ & $<0.1$ & 0.3 & 0.4 & $<0.1$ & $<0.1$ & 0.1 & 0.1 \\
\hline Sc & 10 & 12 & 13 & 9 & 10 & 9 & 7 & 8 \\
\hline Sn & 197 & 215 & 512 & 739 & 230 & 194 & 163 & 140 \\
\hline $\mathrm{Sr}$ & 15.8 & 49.5 & 500.7 & 714.2 & 51.5 & 83.1 & 114.7 & 103.3 \\
\hline $\mathrm{Ta}$ & 0.5 & 1.4 & 1.3 & 0.8 & 1.2 & 0.8 & 0.8 & 0.9 \\
\hline Th & 1.8 & 24.1 & 26.8 & 18.3 & 5.0 & 7.1 & 16.2 & 72.7 \\
\hline $\mathrm{U}$ & 2.4 & 4.8 & 11.2 & 8.1 & 4.0 & 1.6 & 1.6 & 4.4 \\
\hline V & 44 & 55 & 68 & 58 & 40 & 34 & 31 & 38 \\
\hline $\mathrm{Zn}$ & 14 & 14 & 12 & 4 & 18 & 24 & 21 & 21 \\
\hline $\mathrm{Zr}$ & 112.3 & 206.3 & 209.4 & 178.3 & 186.5 & 69.2 & 44.7 & 58.9 \\
\hline $\mathrm{La}$ & 5.0 & 56.1 & 53.0 & 61.0 & 11.8 & 11.5 & 21.9 & 84.2 \\
\hline Ce & 16.3 & 111.4 & 107.1 & 112.2 & 30.2 & 25.5 & 43.4 & 155.5 \\
\hline $\operatorname{Pr}$ & 2.86 & 12.01 & 12.49 & 12.36 & 4.80 & 3.36 & 5.09 & 15.57 \\
\hline $\mathrm{Nd}$ & 15.3 & 41.5 & 44.1 & 45.0 & 22.2 & 13.6 & 19.4 & 51.2 \\
\hline Sm & 5.22 & 8.27 & 9.57 & 9.06 & 6.70 & 4.28 & 4.00 & 8.48 \\
\hline $\mathrm{Eu}$ & 3.31 & 5.63 & 6.07 & 12.21 & 4.27 & 2.41 & 2.00 & 3.24 \\
\hline $\mathrm{Tb}$ & 1.09 & 1.81 & 1.59 & 1.83 & 1.32 & 0.99 & 0.69 & 1.25 \\
\hline Gd & 5.74 & 10.10 & 8.91 & 9.62 & 7.04 & 5.09 & 4.28 & 7.30 \\
\hline Dy & 6.76 & 10.77 & 9.58 & 12.20 & 8.99 & 6.56 & 4.21 & 7.38 \\
\hline Но & 1.33 & 2.00 & 1.84 & 2.96 & 1.94 & 1.28 & 0.81 & 1.54 \\
\hline $\mathrm{Er}$ & 3.84 & 5.78 & 5.93 & 8.93 & 5.81 & 3.95 & 2.38 & 4.79 \\
\hline $\mathrm{Tm}$ & 0.54 & 0.94 & 0.97 & 1.42 & 0.78 & 0.59 & 0.35 & 0.71 \\
\hline $\mathrm{Yb}$ & 3.82 & 4.67 & 5.23 & 7.87 & 5.20 & 3.81 & 1.90 & 4.41 \\
\hline $\mathrm{Lu}$ & 0.48 & 0.64 & 0.72 & 1.06 & 0.68 & 0.55 & 0.28 & 0.62 \\
\hline $\mathrm{Eu} / \mathrm{Eu}^{*}$ & 1.85 & 1.88 & 2.01 & 4.00 & 1.90 & 1.58 & 1.48 & 1.26 \\
\hline $\mathrm{La}_{\mathrm{N}} / \mathrm{Yb}_{\mathrm{N}}$ & 0.88 & 8.10 & 6.83 & 5.23 & 1.53 & 2.03 & 7.77 & 12.87 \\
\hline $\mathrm{La}_{\mathrm{N}} / \mathrm{Sm}_{\mathrm{N}}$ & 0.60 & 4.27 & 3.48 & 4.24 & 1.11 & 1.69 & 3.44 & 6.25 \\
\hline इREE & 71.59 & 271.62 & 267.10 & 297.72 & 111.73 & 83.47 & 110.69 & 346.19 \\
\hline
\end{tabular}

Drahota et al. 2005); however, amphiboles in ironrich skarns are alkali-poor, usually ferro-hornblende to ferro-actinolite (Němec 1968, 1970). K-rich hastingsite to potassic-hastingsite with slightly lower $\mathrm{K}_{2} \mathrm{O}$ is present in some of the pegmatites cross-cutting the Vlastějovice skarn (Žáček 2007; Novák et al. 2013).

Compositions of garnet (Ca-rich almandine) in the Vevčice skarn (types A, C) and in the diorite pegmatite are nearly identical. The observed compositional range is significantly different from that found in garnet from amphibole-bearing pegmatites at Vlastějovice with grossular-andradite containing low spessartine and no pyrope components (Fig. 4). It likely formed due to contamination by the host skarn (Novák et al. 2013). The microtextural evidence indicates formation of garnet followed by crystallization of amphibole during decompression of the skarns. 


\subsection{Chemical variability of skarn types and relationship to diorite pegmatite}

Origin of skarns of the Moldanubian Zone was interpreted in two contrasting ways:

1) Open-system, metasomatism affecting carbonate rocks (e.g., Němec 1991; Žáček 1997; Bubal 2013);

2) Closed-system, regional metamorphism of Fe-rich protoliths (e. g. Vrána 1987; Pertold et al. 1997; Drahota et al. 2005). The protoliths of skarns in the studied part of the Moldanubian Zone are currently interpreted as originally Fe-rich, mixed rocks of detrital and exhalative origin deposited on the sea floor (Pertoldová et al. 2009). The typical REE patterns of Vevčice skarns are characterized by fractionated LREE, positive Eu anomaly and flat HREE segments. Similar trends are known from submarine hydrothermal exhalative ore deposits (e.g. black smoker chimneys; Hongo and Nozaki 2001; Hongo et al. 2007; Pertoldová et al. 2009). Hydrothermal fluids from seafloor hydrothermal vents typically show LREE enrichment with positive Eu anomalies (e.g. Michard and Albarède 1986; Michard 1989; Klinkhammer et al. 1994; Mills and Elderfield 1995). The REE patterns in the skarns (particularly in the type B) could be interpreted as a pre-metamorphic signature of protolith.

The results of Pertold et al. (1997) from skarns in the Gföhl Unit (Rešice and Slatina) indi-

Fig. 9a - Chondrite-normalized REE-patterns (Boynton 1984) for skarns and pegmatites. $\mathbf{b}$ - Primitive mantle-normalized multi-element patterns of the skarns and diorite pegmatites (normalizing values are from McDonough and Sun 1995). Grey region is the compositional range of migmatites from the Gföhl Unit (Hasalová et al. 2008).
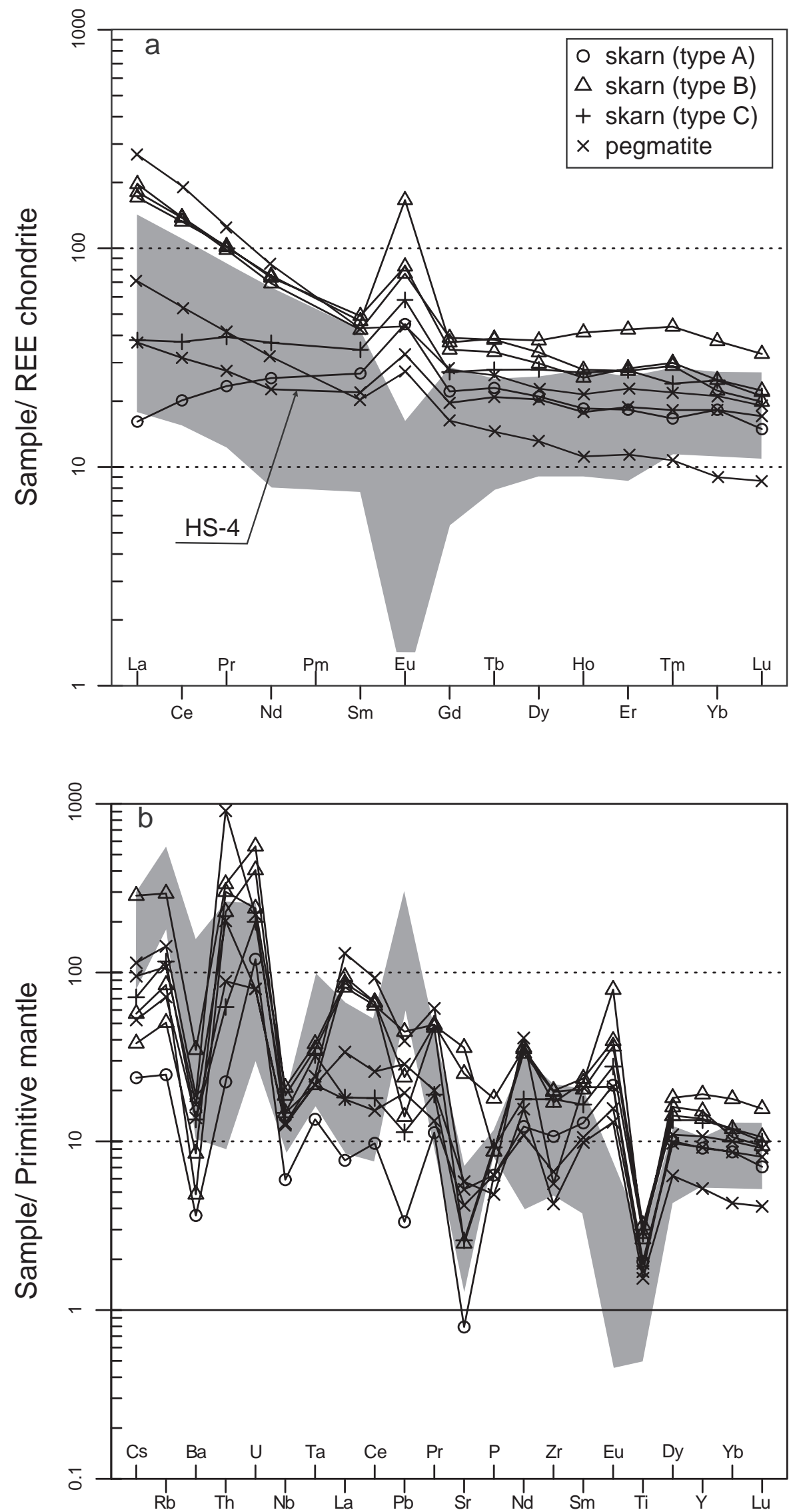

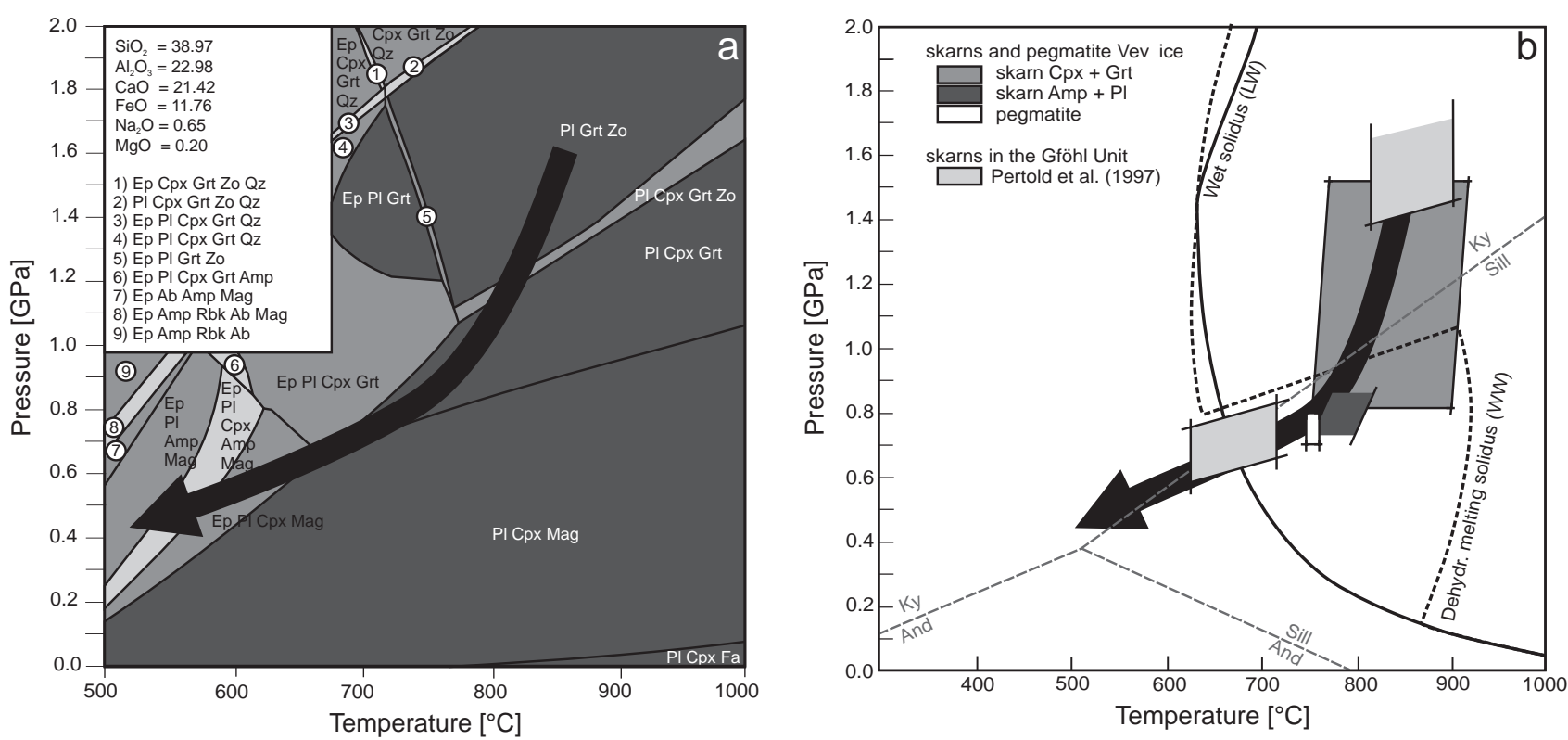

Fig. 10a - P-T pseudosection calculated for the modified composition of skarn HS-2a (Tab. 6) with the PERPLE X software (see text). Dark arrow shows a possible P-T path for the skarns in the Gföhl Unit. b - Pressure-temperature diagram illustrating equilibria for the estimated assemblages of the skarns and diorite pegmatites. The solid line represents the fluid-saturated basalt solidus (Lambert and Wyllie 1972, LW) and dashed curve dehydration melting of amphibolite (Wyllie and Wolf 1993, WW). P-T conditions for Cpx + Grt skarn calculated on the basis of garnet-clinopyroxene geothermometer (Krogh Ravna 2000b) and garnet-clinopyroxene geobarometer (Simakov 2008); T for the skarn Amp + Pl obtained on the basis of amphibole-plagioclase thermometer (Holland and Blundy 1994) for P = 0.8 GPa; P-T conditions for pegmatite calculated on the basis of amphibole-plagioclase thermometer (Holland and Blundy 1994) and Al-in-hornblende geobarometer (Anderson and Smith 1995).

cate that the majority of REE is concentrated in the main rock-forming minerals (pyroxene, garnet and magnetite). Positive Eu anomaly is typical mainly of garnet and pyroxene (magnetite shows variably positive or negative Eu anomalies). Garnet is enriched in HREE and depleted in LREE, in contrast to clinopyroxene and magnetite which show slight enrichment in LREE (Pertold et al. 1997). The LREE are also hosted in accessory minerals such as allanite and titanite; for example, the concentrations $3.4-3.5$ wt. \% and $0.15-0.18$ wt. \% Ce (Tab. 4) in allanite and titanite respectively. The LREEpoor pattern of the sample of garnet-rich skarn (type A) can be therefore explained by its nearly monomineral composition, because Ca-garnet (andradite-grossular) in skarns is often characterized by strong positive $\mathrm{Eu}$ anomaly, depletion in HREE, and enrichment in LREE, in contrast to Al-garnets from metapelites or amphibolites (Gaspar et al. 2008). Observed differences in LREE contents in individual whole-rock analyses of the type B and skarns $\mathrm{C}$ agree with variable contents of allanite and titanite in the studied samples. In particular, skarn type B samples with high degree of LREE fractionation are characterized by higher contents of allanite and titanite compared to other skarn types.

The REE pattern of pegmatite (sample HS-4) is similar to that of the amphibole-rich skarn (sample HS-5; $\left.\mathrm{Ce}_{\mathrm{N}} / \mathrm{Yb}_{\mathrm{N}}=1.5\right)$ except for a weak LREE fractionation. The other two samples of pegmatite show a higher de- gree of LREE fractionation $\left(\mathrm{Ce}_{\mathrm{N}} / \mathrm{Yb}_{\mathrm{N}}=5.9-9.1\right)$. These results can be explained by variable modal amounts of amphibole, allanite and titanite in the pegmatite samples.

\subsection{Evolution of the skarn mineral assemblages from the Gföhl Unit}

Based on earlier published data and the results of this work, we can identify several stages of metamorphic evolution of skarns from the Gföhl Unit, with one exception including the Vevčice skarn body:

The first stage is represented by relics of hydrous prepeak skarn mineral assemblages present in the skarns of the entire Moldanubian Zone. However, they are only preserved as rare inclusions in garnet and clinopyroxene. Garnet often contains epidote inclusions (Pertoldová et al. 2009), whereas amphibole inclusions are rare. Clinopyroxene from the skarns in the Moldanubian Zone sometimes contains inclusions of the hisingerite-neotocite group minerals (Drahota et al. 2005). Specific features of the PERPLE_X pseudosection for skarn sample HS-2a (Fig. 10a) are: (1) the occurrence of amphibole at $\mathrm{T} \leq$ $625^{\circ} \mathrm{C}$, (2) the appearance of the mineral assemblage Grt $+\mathrm{Cpx}+\mathrm{Pl}$ at P $>0.6 \mathrm{GPa}$ and $\mathrm{T}>625^{\circ} \mathrm{C}$, and (3) the appearance of quartz at high $\mathrm{P}$ and low $\mathrm{T}$.

Second stage of metamorphic evolution is documented by rare jadeite-rich (up to $22 \mathrm{~mol}$. \%) clinopyroxene in- 
clusions in garnet; this HP mineral assemblage (1.3-1.4 GPa at $790-850^{\circ} \mathrm{C}$; Pertold et al. 1997) is known from a few skarns bodies only (Rešice, Slatina) in vicinity of the Vevčice occurrence. Complete disappearance of the Na-rich clinopyroxene has resulted in the formation of a new mineral assemblage with Na-rich plagioclase and Al-rich hedenbergite. However, evidence for this stage in the form of jadeite-rich pyroxene was never observed in the Vevčice skarn.

Third stage is represented by the mineral assemblage including garnet, hedenbergite, plagioclase, quartz, magnetite, with minor epidote, titanite and allanite. In Vevčice, we have calculated the $\mathrm{P}-\mathrm{T}$ conditions of $734-823^{\circ} \mathrm{C}$ at $0.9-1.5 \mathrm{GPa}$ based on the garnet-clinopyroxene geothermometer (Krogh and Ravna 2000a) and garnet-clinopyroxene geobarometer (Simakov 2008). These conditions document post-peak recrystallization during exhumation and are in a good agreement with the estimates previously published for other skarns in the Gföhl Unit (Pertold et al. 1997). Partial melting in the surrounding Gföhl gneiss started within this $\mathrm{P}-\mathrm{T}$ range ( $790^{\circ} \mathrm{C}$ and $0.9 \mathrm{GPa}$; Hasalová et al. 2008).

At the same time the pressures of 0.9-1.0 GPa at temperature $\sim 800^{\circ} \mathrm{C}$ are very close to amphibole dehydration melting solidus of basic rocks (Wyllie and Wolf 1993). Temperature of the pegmatite melt formation at Vevčice could have been above $\sim 800^{\circ} \mathrm{C}$ when considering zircon saturation temperature of $c .820^{\circ} \mathrm{C}$ (calculated after Watson and Harrison 1983).

Fourth stage is represented by a mineral assemblage including amphibole and epidote; it formed at $763-808^{\circ} \mathrm{C}$ at $0.8 \mathrm{GPa}$ based on the amphibole and plagioclase thermometer (Holland and Blundy 1994). The similar chemical composition of amphiboles from the skarns and diorite pegmatite can be interpreted as extensive mid-crustal re-equilibration of the skarn mineral assemblage of the third stage.

\subsection{Conditions of pegmatite crystallization}

Mineral assemblage of the pegmatites at Vevčice $(\mathrm{Pl}+$ Amp \pm Grt \pm Qz \pm Aln \pm Mag) allowed estimation of crystallization $\mathrm{P}-\mathrm{T}$ conditions; textural relationships indicate simultaneous crystallization of amphibole, plagioclase and garnet. Solidification temperatures were calculated from the amphibole-plagioclase thermometer of Holland and Blundy (1994) in combination with garnet-amphibole thermometer (Krogh and Ravna 2000b), and the pressure was obtained from Al-in-hornblende barometer (Anderson and Smith 1995). The estimated P-T conditions (Tab. 8) are $747-758^{\circ} \mathrm{C}$ and $0.7-0.8$ $\mathrm{GPa}$, in agreement with the pressure stability range of magmatic epidote (over 0.5 to 3.0 GPa; Poli and Schmidt 1995,2004 ) and with the maximum temperature stability limit for magmatic epidote under fluid-saturated condi-
Tab. 8 Calculated P-T conditions for selected mineral assemblages of the Vevčice skarn and diorite pegmatite

\begin{tabular}{lcc}
\hline & $\mathrm{T}\left({ }^{\circ} \mathrm{C}\right)$ & $\mathrm{P}(\mathrm{GPa})$ \\
\hline skarn type A & $\mathbf{C p x}+\mathbf{G r t}+\mathbf{P l}+\mathbf{Q z}$ & \\
$\mathrm{T}($ Grt-Cpx $) / \mathrm{P}(\mathrm{Grt}-\mathrm{Cpx} 1)$ & $759-910$ & $0.8-1.5$ \\
$\mathrm{~T}($ Grt-Cpx)/P(Grt-Cpx2) & $773-918$ & $0.7-1.5$ \\
skarn type C & $\mathbf{A m p}+\mathbf{G r t}+\mathbf{P l}+\mathbf{Q z}+\mathbf{E p}$ & \\
$\mathrm{T}($ Amp/Pl) & $763-808$ & at 0.8 \\
pegmatite & $\mathbf{A m p}+\mathbf{G r t}+\mathbf{P l}+\mathbf{E p}+\mathbf{Q z}$ & \\
$\mathrm{T}(\mathrm{Zr})$ & 821 & - \\
$\mathrm{T}(\mathrm{Grt}-\mathrm{Amp})$ & $754-758$ & - \\
$\mathrm{T}($ Amp/Pl)/P(Amp) & $747-755$ & $0.7-0.8$ \\
\hline
\end{tabular}

$\mathrm{T}($ Grt-Cpx) = garnet-clinopyroxene geothermometer (Krogh Ravna 2000b); P(Grt-Cpx1) garnet-clinopyroxene geobarometer (Simakov 2008); P(Grt-Cpx2) garnet-clinopyroxene geobarometer (Mukhopadhyay 1991); $\mathrm{T}(\mathrm{Zr})$ = zircon saturation geothermometer (Watson and Harrison 1983); $\mathrm{T}(\mathrm{Grt}-\mathrm{Amp})$ = garnet-amphibole geothermometer (Krogh Ravna 2000a); T(Amp-Pl) = amphibole-plagioclase geothermometer (Holland and Blundy 1994), P(Amp) = Al-in-hornblende geobarometer (Anderson and Smith 1995)

tions in tonalite $\left(\sim 750^{\circ} \mathrm{C}\right.$; Poli and Schmidt 2004). Experimental studies (Holdaway 1972; Liou 1973) also indicated a strong dependence of epidote thermal stability on oxygen fugacity. The observed presence of the quartz + titanite (with inclusions of magnetite and ilmenite) assemblage requires low $f\left(\mathrm{O}_{2}\right)$, slightly above the QFM buffer (Wones 1989). The P-T conditions of the pegmatite melt crystallization $\left(747-758^{\circ} \mathrm{C}\right.$ and $\left.0.7-0.8 \mathrm{GPa}\right)$ are $\sim 60-100^{\circ} \mathrm{C}$ higher than the fluid-saturated solidus of basaltic system (Lambert and Wyllie 1972, $\sim 650^{\circ} \mathrm{C}$ at $0.8 \mathrm{GPa}$; Fig. 10b).

For similar pegmatites with fluorite and amphibole intruding the skarn near Vlastějovice were obtained slightly lower P-T conditions of crystallization $\left(600-640^{\circ} \mathrm{C}\right.$ and 0.4-0.6 GPa; Ackerman et al. 2007).

\subsection{Source of melt}

Formation of the diorite pegmatite melt can be explained by three main mechanisms:

(a) fluid-present melting of relatively basic protoliths with, or without, metasomatic reaction between the host skarn and external fluids ( $\mathrm{Si}, \mathrm{Na}, \mathrm{Ba}, \mathrm{Sr}, \mathrm{K}, \mathrm{H}_{2} \mathrm{O}$-rich; e.g. Pattison 1991),

(b) in-situ dehydration melting of basic igneous rocks (e.g. Johnson et al. 2012 and the references therein),

(c) injection by acidic melt from a more distal source and its contamination by the host skarn (e.g., Novák et al. 2012, 2013; Gadas et al. 2014).

\subsubsection{Fluid-present melting and/or metasomatic reaction}

Addition of $\mathrm{H}_{2} \mathrm{O}$ to the basic protolith (including skarns) reduces thermal stability of the mineral assemblage (e.g. 
Green 1982). Fluid-present melting of basic rocks at pressure exceeding $1.5 \mathrm{GPa}$ typically generates adakitic melts $\left(\mathrm{SiO}_{2}>56\right.$ wt. \%, $\mathrm{Al}_{2} \mathrm{O}_{3}>15$ wt. \%, $\mathrm{Na}_{2} \mathrm{O}>5$ wt. \%, high $\mathrm{Sr} / \mathrm{Y}>20-40$, high HFSE and low HREE) and the garnet + clinopyroxene \pm amphibole residue (e.g. Defant and Drummond 1990; Peacock et al. 1994; Rapp and Watson 1995; Winther 1996; Drummond et al. 1996; Xiong et al. 2005, 2006; Moyen 2009). However, the studied pegmatite has a chemical composition close to diorite and gabbro with lower contents of $\mathrm{Na}_{2} \mathrm{O}$ (2.9-3.6 wt. \%), $\mathrm{SiO}_{2}(46.2-56.1$ wt. \%) and very low $\mathrm{Sr} / \mathrm{Y}$ ratio (2.1-5.1).

\subsubsection{Dehydration melting of skarn and effects of fractional crystallization}

Garnet zoning in skarns from several localities of the Gföhl Unit reflects post-peak decompression from 1.2 $\mathrm{GPa}$ at $c .800^{\circ} \mathrm{C}$ to $0.6-0.8 \mathrm{GPa}$ and $670^{\circ} \mathrm{C}$ (Drahota et al. 2005). These conditions would be appropriate for partial melting of a wide range of basic rocks (Fig. 10b) including hydrous mineral assemblages of skarns. Similar P-T path was also assumed for skarns from the southeastern part of the Gföhl Unit, which were affected by an analogous HT-HP metamorphic event $\left(800^{\circ} \mathrm{C}\right.$ and $>1.4 \mathrm{GPa}$ ) followed by partial re-equilibration under amphibolite-facies conditions (Pertold et al. 1997).

The P-T conditions calculated for the formation of garnet-rich metamorphic assemblages in skarns (Pertold et al. 1997) exceed the stability field of epidote and amphibole (Vielzeuf and Schmidt 2001; Qian and Hermann 2013). During dehydration melting reactions, epidote and/ or amphibole break down to garnet and/or clinopyroxene (Poli 1993; Vielzeuf and Schmidt 2001). Experimental studies on the dehydration-melting of amphibole-rich rocks produced melt with wide range of chemical compositions according to the protolith types and P-T conditions of melting (Hacker 1990; Skjerlie and Johnston 1992; Wolf and Wyllie 1994; Watkins et al. 2007).

Partial melting of Amp + Pl pair occurs more likely as a result of high-pressure ( $\mathrm{P}>1 \mathrm{GPa}$ ) reaction Amp $+\mathrm{Pl}=\mathrm{Ep} \pm \mathrm{Grt}+\mathrm{Cpx}+$ melt, rather than due to the medium-pressure $(\mathrm{P}<1 \mathrm{GPa})$ reaction $\mathrm{Amp}+\mathrm{Pl}+\mathrm{Qz}=$ Cpx + melt (López and Castro 2001; Zhang et al. 2014). Another possible melting reaction involving epidote/ zoisite is Amp + Ep/Zo + Qz = Grt + melt (Vielzeuf and Schmidt 2001).

The $\mathrm{P}-\mathrm{T}$ conditions for peak mineral assemblages in the Gföhl Unit skarns $\left(1.3-1.4 \mathrm{GPa}\right.$ at $790-850^{\circ} \mathrm{C}$; Pertold et al. 1997) are consistent with the formation of tonalitic to trondhjemitic melt (e.g. Rushmer 1991; Wyllie and Wolf 1993). Petrographically and chemically similar pegmatites $(\mathrm{Pl}+\mathrm{Cpx} \pm \mathrm{Opx} \pm \mathrm{Amp} \pm$ Qz; Figs $7-8)$ were described as products of partial melting of metagabbros under granulite-facies conditions (Pattison 1991; Berger et al. 2008; Johnson et al. 2012). Moreover, Vevčice pegmatite contains angular to subangular fragments of garnet-rich skarn (type A), which show mineralogical and chemical similarity with solid product (restite) of dehydration melting (López and Castro 2001; Zhang et al. 2014).

Discontinuous veins of diorite pegmatite are oriented randomly to the foliation and compositional layering of the host skarns. Diffuse leucocratic rims are sometimes present at the contact of pegmatite and garnet skarn xenoliths. Brecciation of skarn layers demonstrates brittle deformation during melt segregation. Amphibole-rich xenoliths contain relics of clinopyroxene and can be interpreted as products of peritectic reaction between clinopyroxene skarn and pegmatite melt.

Genesis of anatectic melt requires operation of melting reactions driven by changing $\mathrm{P}-\mathrm{T}$ conditions, and segregation of the melt assisted by local stress field (Sawyer 1991). Chemical composition of the segregated melt can be strongly affected by equilibration with the host rocks (e.g. Sawyer 1991; Bea 1996) but can be also modified during its own evolution (fractional crystallization). Melt would migrate into decimetre- to metre-scale channels; they are now represented by the pegmatite veins, providing a record of deformation-controlled melt migration contemporary with the melt formation (e.g., Brown et al. 2011). Chemical composition of diorite pegmatite veins is inconsistent with the results of experimental works (Fig. 7a-b; Hacker 1990; Skjerlie and Johnston 1992; Wolf and Wyllie 1994; Watkins et al. 2007). The major differences between studied diorite pegmatite and results of experimental works are low $\mathrm{SiO}_{2}$ (46-56 vs. 52-80 wt. \%) and high $\mathrm{Fe}_{2} \mathrm{O}_{3}$ (11.2-17.5 vs. 0.3-8.3 wt. \%). The formation of analogous (Fig. 8) unusual pegmatites was interpreted by Johnson et al. (2012) as a result of magmatic fractionation. The large modal abundances of amphibole and plagioclase and the small amount of the quartz and K-feldspar suggest that the Vevčice pegmatites can be amphibole and plagioclase cumulates (Johnson et al. 2012).

\subsubsection{Injection and/or contamination of acidic melt from a more distal source}

Diorite pegmatites are also known from other skarn bodies in the region. For instance, from those at Rešice or Slatina Pertold et al. (1997) described several mm thick felsic veinlets with a Pl + Kfs + Amp + Ti-rich Mgt mineral assemblage. Texturally and mineralogically homogeneous veins are not typical of anatectic melts modified by assimilation of surrounding rocks (Novák et al. 2012; Gadas et al. 2014). On the other hand, diffusive exchange of elements between magma 
and country-rock occurs at extremely rapid rates (e.g. Erdmann et al. 2007).

Pertold et al. (1997) reported that the banding of the nearby Rešice skarn body is cross-cut by veins of biotite leucogranite and similar veins of diatexite to biotite granite are also present at the border of the Vevčice skarn outcrop. This melt derived from the surrounding migmatised Gföhl gneisses coexisted with the skarn without any significant changes to the modal composition (biotite is present as the only ferromagnesian mineral). This can be explained by the multiple injections of anatectic melt during skarn exhumation (e.g., young dykes would have undergone little or no contamination as a consequence of low ambient temperature).

Formation of the diorite pegmatite could have resulted from pervasive infiltration of a felsic melt along grain boundaries. Pervasive melt infiltration driven by grain-scale porous flow along high-porosity dissolution channels (e.g. McKenzie 1989; Kelemen et al. 1997; Reiners 1998) cannot be excluded, leading to the reaction: amphibole (skarn) + granitic melt $\rightarrow$ dioritic to gabbroic melt + clinopyroxene + garnet. The pegmatites in the variation diagrams are usually situated between the compositions of the dominant type A skarns and the Gföhl gneisses (Fig. 8). Indeed, if compared to Gföhl gneisses affected by high degree of partial melting (nebulites; Hasalová et al. 2008), the studied diorite pegmatites are characterized by higher $\mathrm{Al}_{2} \mathrm{O}_{3}, \mathrm{Na}_{2} \mathrm{O}, \mathrm{CaO}$, $\mathrm{FeO}_{\mathrm{t}}$, MgO, REE, Th and lower $\mathrm{Zr}, \mathrm{Rb}, \mathrm{P}_{2} \mathrm{O}_{5}$ and $\mathrm{K}_{2} \mathrm{O}$ contents (Figs 8-9). Theoretically, the contaminated melt would be enriched mainly in incompatible elements such as HFSE and depleted in alkalis (e.g., Rapp and Watson 1995; Winther 1996; Xiong et al. 2005, 2006). In reality, the studied diorite pegmatites are depleted in $\mathrm{Rb}$ and $\mathrm{K}_{2} \mathrm{O}$, but content of numerous HFSE (e. g., U, $\mathrm{Nb}, \mathrm{Y}$ ) is similar as in leucosome generated by partial melting of the surrounding Gföhl gneisses (Fig. 8).

Based on the presented data we are not able to offer a definite conclusion here. However, our results slightly favour scenario that pegmatites could be a product of interaction between skarns and anatectic melts derived from the surrounding Gföhl gneisses affected by high degree of partial melting. Some differences (e. g., high content of $\mathrm{Na}_{2} \mathrm{O}$ and low contents of $\mathrm{Zr}, \mathrm{P}_{2} \mathrm{O}_{5}$ in comparison to the skarns and Gföhl gneisses) can be explained if the composition of the contaminated magma was changed by subsequent fractional crystallization and/or crystal accumulation (Johnson et al. 2012).

\section{Conclusions}

Clinopyroxene \pm garnet \pm amphibole \pm magnetite \pm plagioclase \pm quartz skarn is a characteristic rock type of the Gföhl Unit, including the Vevčice locality in S Moravia. Small skarn bodies (usually 20-30 m in diameter) are locally cross-cut by numerous veins of diorite pegmatites or the melt patches. They are coarse-grained but do not show zoning and textures typical of granitic pegmatites. Characteristic is the lack of unidirectional solidification textures, graphic and blocky zones, as well as, in most cases, any reaction zones at the contact with the host skarn. Major pegmatite minerals are potassichastingsite (0.3-0.5 apfu K), plagioclase $\left(\mathrm{An}_{15-44}\right)$, and garnet $\left(\mathrm{Alm}_{52-60} \mathrm{Grs}_{25-37} \mathrm{Adr}_{5-7} \operatorname{Prp}_{3-7} \mathrm{Sps}_{1-3}\right)$, whereas minor minerals are represented by $\mathrm{K}$-feldspar (locally enclosed in plagioclase), quartz, allanite-epidote, clinopyroxene, and accessory titanite. Amphibole-rich diorite pegmatite with low silica contents $\left(\mathrm{SiO}_{2} 46-56\right.$ wt. \%) cutting the Vevčice skarn probably formed as a product of partial melt infiltration from surrounding migmatites and subsequent magma contamination by the country-rock skarn, with or without attendant fractional crystallization/crystal accumulation. Similarity in composition of amphiboles in the skarn and in the pegmatite can indicate formation under comparable conditions as were those estimated for the pegmatite crystallization $\left(c .770^{\circ} \mathrm{C}\right.$ and $\left.0.7-0.8 \mathrm{GPa}\right)$.

Acknowledgements. The authors wish to thank J. Cempírek for comments on the early version of the manuscript and for its translation into English. We thank also Radek Škoda and Petr Gadas for the electron microprobe work. This work was supported by the Czech Geological Survey research project No. 321180 to DB and financial funds to the Moravian Museum by the Ministry of Culture of the Czech Republic as part of its long-term conceptual development programme for research institutions (ref. MK000094862) (HS). The research of LK was financially supported by project No. RVO67985831 of the Institute of Geology of the Czech Academy of Sciences, v. v. i., Prague and the BUT project No. LO1408 "AdMaS UP - Advanced Materials, Structures and Technologies", supported by the Ministry of Education, Youth and Sports of the Czech Republic under the "National Sustainability Programme I". We acknowledge valuable comments of D. Dolejš and M. Götzinger, as well as the editor V. Kachlík.

\section{References}

Ackerman L, Zachariáš J, Pudilová M (2007) P-T and fluid evolution of barren and lithium pegmatites from Vlastějovice, Bohemian Massif, Czech Republic. Int J Earth Sci (Geol Rundsch) 96: 623-638

Anderson J, Smith D (1995) The effect of temperature and oxygen fugacity on Al-in-hornblende barometry. Amer Miner 80: 549-559 
BEA F (1996) Residence of REE, Y, Th and U in granites and crustal protoliths: implications for the chemistry of crustal melts. J Petrol 37: 521-552

Berger A, Burri T, Alt-Epping P, Engi M (2008) Tectonically controlled fluid flow and water-assisted melting in the middle crust: an example from the Central Alps. Lithos 102: 598-615

Boynton WV (1984) Cosmochemistry of the rare earth elements: meteorite studies. In: HENDERson P (ed) Rare Earth Element Geochemistry. Elsevier, Amsterdam, pp 63-114

Brown M, Korhonen FJ, Siddoway CS (2011) Organizing melt flow through the crust. Elements 7: 261-266

Bubal J (2013) Geochemistry and Origin of Skarns of the Bohemian Massif. Unpublished MSci thesis, Charles University, Prague, pp 1-147 (in Czech with English abstract)

Cempírek J, Houzar S, Novák M (2008) Complexly zoned niobian titanite from hedenbergite skarn at Písek, Czech Republic, constrained by substitutions $\mathrm{Al}(\mathrm{Nb}, \mathrm{Ta})$ $\mathrm{Ti}_{-2}, \mathrm{Al}(\mathrm{F}, \mathrm{OH})(\mathrm{TiO})_{-1}$ and $\mathrm{SnTi}_{-1}$. Mineral Mag 76: 1293-1305

Connolly JAD (2005) Computation of phase equilibria by linear programming: a tool for geodynamic modeling and its application to subduction zone decarbonation. Earth Planet Sci Lett 236: 524-541

Connolly JAD, Cesare B (1993) C-O-H-S fluid composition and oxygen fugacity in graphite metapelites. J Metamorph Geol 11: 379-388

CoOKe RA, O’Brien PJ (2001) Resolving the relationship between high $\mathrm{P}-\mathrm{T}$ rocks and gneisses in collisional terranes: an example from the Gföhl gneiss-granulite association in the Moldanubian Zone, Austria. Lithos 58: 33-54

Defant MJ, Drummond MS (1990) Derivation of some modern arc magmas by melting of young subducted lithosphere. Nature 347: 662-665

Drahota P, Pertold Z, Pudilová M (2005) Three types of skarn in the northern part of the Moldanubian Zone, Bohemian Massif - implications for their origin. J Czech Geol Soc 50: 19-33

Drummond MS, Defant MJ, Kepezhinskas PK (1996) Petrogenesis of slab-derived trondhjemite-tonalite-dacite/adakite magmas. Trans Roy Soc Edinb, Earth Sci 87: 205-215

Dudek A, MatĚJovsKá O, SuK M (1974) Gföhl orthogneiss in the Moldanubicum of Bohemia and Moravia. Krystalinikum 10: 67-78

Erdmann S, London D, Morgan GB VI, Clarke DB (2007) The contamination of granitic magma by metasedimentary country-rock material: an experimental study. Canad Mineral 45: 43-61

Fiala J, Fuchs G, Wendt JI (1995) Stratigraphy of the Moldanubian Zone. In: Dallmeyer RD, Franke W, WeBER K (eds) Pre-Permian Geology of Central and Eastern Europe. Springer, Berlin, pp 417-428
Filip J, Houzar S, Sulovský P (2002) Allanite and its decay products in the skarn and pegmatite from Rešice. Acta Mus Moraviae, Sci Geol 87: 87-101 (in Czech with English abstract)

Friedl G, Finger F, Paquette JL, von Quadt A, McNaughton NJ, Fletcher IR (2004) Pre-Variscan geological events in the Austrian part of the Bohemian Massif deduced from U/Pb zircon ages. Int J Earth Sci 93: 802-823

FrITz H (1995) The Raabs Series, a probable Variscan suture in the SE Bohemian Massif. Jb Geol B-A 138: 639-653

Fuchs G, Matura A (1976) Zur Geologie des Kristallins der südlichen Böhmischen Masse. Jb Geol B-A 119: 1-43

Gadas P, Novák M, Cempírek J, Filip J, Vašinová Galiová M, GroAt LA, VšiAnskÝ D (2014) Mineral assemblages, compositional variation and crystal structure of feruvitic tourmaline from contaminated anatectic pegmatite in Mirošov near Strážek, Moldanubian Zone, Czech Republic. Canad Mineral 52: 285-301

Gaspar M, KnaAck C, Meinert LD, Moretti R (2008) REE in skarn systems: a LA-ICP-MS study of garnets from the Crown Jewel gold deposit. Geochim Cosmochim Acta 72: 185-205

GolıÁš V (2002) Thorium occurrences in the Czech Republic and their mineralogy. In: KŘíBEK B, ZEMAN J (eds) Uranium Deposits - From Their Genesis to the Environmental Aspects. Czech Geological Survey, Prague, pp 53-56

GreEn TH (1982) Anatexis of mafic crust and high pressure crystallization of andesite. In: THORPE RS (ed) Andesites. Wiley, Chichester, pp 465-487

Gulyaeva TY, Gorelikova NV Karabtsov AA (1986) High potassium-chlorine-bearing hastingsites in skarn from Primorye, Far East USSR. Mineral Mag 50: 724-728

Hacker BR (1990) Amphibolite-facies-to-granulite-facies reactions in experimentally deformed, unpowdered amphibolite. Amer Miner 75: 1349-1361

Hasalová P, Janoušek V, Schulmann K, ŠTíPská P, ERBan V (2008) From orthogneiss to migmatite: geochemical assessment of the melt infiltration model in the Gföhl Unit (Moldanubian Zone, Bohemian Massif). Lithos 102: 508-537

HoldaWAY MJ (1972) Thermal stability of Al-Fe-epidote as a function of $\mathrm{fO}_{2}$ and $\mathrm{Fe}$ content. Contrib Mineral Petrol 37: 307-340

Holland T, Blundy J (1994) Non-ideal interactions in calcic amphiboles and their bearing on amphibole-plagioclase thermometry. Contrib Mineral Petrol 116: 433-447

Holland TJB, Powell R (1998) An internally consistent thermodynamic data set for phases of petrological interest. J Metamorph Geol 16: 309-43

Homola V, Müller K, Smrkovská V (1968) Geologischgeophysikalische Studien im Gebiet des westmährischen Moldanubikums. Freiberg Forsch H C C 227: 1-71 
Hongo Y, NoZAKI Y (2001) Rare earth element geochemistry of hydrothermal deposits and Calyptogena shell from the Iheya Ridge vent field, Okinawa Trough. Geochem J 35: 347-354.

Hongo Y, Obata H, Gamo T, Nakasema M, Ishibashi J, Konno U, Saegusa S, Ohkubo S, Tsunogai U (2007) Rare earth elements in the hydrothermal system at Okinawa Trough back-arc basin. Geochem J 41: 1-15

HouZAR S, ŠMERDA J, BuRIÁNEK D (2014) A new occurrence of skarn in the Gföhl Unit at Vevčice near Jevišovice: the mineral assemblage of skarn and contaminated amphibole-bearing pegmatites. Geol Výzk Mor Slez 20: 62-70 (in Czech with English abstract)

JANOUŠEK V, FARROW CM, ERBAN V (2006) Interpretation of whole-rock geochemical data in igneous geochemistry: introducing Geochemical Data Toolkit (GCDkit). J Petrol 47: 1255-1259

Johnson TE, Fischer S, White RW, Brown M, Rollinson HR (2012) Archaean intracrustal differentiation from partial melting of metagabbro - field and geochemical evidence from the Central Region of the Lewisian Complex, NW Scotland. J Petrol 53: 2115-2138

Kelemen PB, Hirth G, Shimizu N, Spiegelman M, Dick HJB (1997) A review of melt migration processes in the adiabatically upwelling mantle beneath oceanic spreading ridges. Philos Trans Roy Soc London, Ser A 355: 283-318

Klinkhammer GP, Elderfield H, Edmond JM, Mitra A (1994) Geochemical implications of rare earth element patterns in hydrothermal fluids from mid-ocean ridges. Geochim Cosmochim Acta 58: 5105-5113

Krmíček L, Cempírek J, Havlín A, Přichystal A, Houzar S, KrmíčKovÁ M, GADAS P (2011) Mineralogy and petrogenesis of a Ba-Ti-Zr-rich peralkaline dyke from Šebkovice (Czech Republic): recognition of the most lamproitic Variscan intrusion. Lithos 121: 74-86

Krogh Ravna EJ (2000a) Distribution of $\mathrm{Fe}^{2+}$ and $\mathrm{Mg}$ between coexisting garnet and hornblende in synthetic and natural systems: an empirical calibration of the garnet-hornblende $\mathrm{Fe}-\mathrm{Mg}$ geothermometer. Lithos 53: 265-277

Krogh Ravna EJ (2000b) The garnet-clinopyroxene $\mathrm{Fe}^{2+}-$ Mg geothermometer: an updated calibration. J Metamorph Geol 2: 211-219

Kusbach V, Janoušek V, Hasalová P, Schulmann K, FANNING CM, ERBAN V, UlRich S (2015) Importance of crustal relamination in origin of the orogenic mantle peridotite-high-pressure granulite association: example from the Náměšt' Granulite Massif (Bohemian Massif, Czech Republic). J Geol Soc, London 172: 479-490

LAMBert IB, Wyllie PJ (1972) Melting of gabbro (quartz eclogite) with excess water to 35 kilobars, with geological applications. J Geol 80: 693-708
Leake BE, Wooley AR, Arps CES, Birch WD, Gilbert MC, Grice JD, Hawthorne FC, Kato A, Kisch HJ, Krivovichev VG, Linthout K, Laird J, Mandarino J, Maresch WV, Nickel EH, Rock NMS, Schumacher JC, Smith J C, Stephenson NCN, Whittaker EJW, Youzhi G (1997) Nomenclature of amphiboles: report of the Subcommittee on Amphiboles of the International Mineralogical Association Commission on New Minerals and Mineral Names. Mineral Mag 61: 295-321

Liou JG (1973) Synthesis and stability relations of epidote $\mathrm{Ca}_{2} \mathrm{Al}_{2} \mathrm{FeSi}_{3} \mathrm{O}_{12}(\mathrm{OH})$. J Petrol 14: 381-413

López S, CASTRO A (2001) Determination of the fluid-absent solidus curve and supersolidus phase relationships of MORB-derived amphibolites in the range 4-14 kbar. Amer Miner 86: 1396-1403

MARTINeC P (1977) Erlans and skarns in the complex so called Gföhl gneisses in Moldanubicum of south western Moravia. In: Pouba Z (ed) Correlation of Proterozoic and Paleozoic Stratabound Deposits V. Faculty of Science, Charles University, Prague, pp 163-182 (in Czech with English abstract)

Massonne HJ, Willner AP (2008) Dehydration behaviour of metapelites and mid-ocean ridge basalt at very-low to low grade metamorphic conditions. Eur J Mineral 20: 867-879

MatĚJovsKÁ O (1975) The Moldanubian gneiss series of south-western Moravia and its relation to granulites. Věst Ústř Úst Geol 50: 345-351

Matějovská O, Batík P, Dornič J, Havlíček P, Hazdrová M, JenČeK V, Kadlec E, Líbalová J, Šalanský K, Volšan V (1987) Explanations to the geological map of ČSSR $1: 25000$ sheet 34-111 Višňové. Czech Geological Survey, Prague, pp 1-54 (in Czech with English abstract)

Matte P, Maluski H, Rajlich P, Franke W (1990) Terrane boundaries in the Bohemian Massif: result of large-scale Variscan shearing. Tectonophysics 177: 151-170

MazDAB FK (2003) The diversity and occurrence of potassium-dominant amphiboles. Canad Mineral 41: 1329-1344

McDonough WF, Sun SS (1995) The composition of the Earth. Chem Geol 120: 223-253

McKenzie D (1989) Some remarks on the movement of small melt fractions in the mantle. Earth Planet Sci Lett 95: 53-72

Medaris G JR, Fournelle JH, Ghent ED, Jelínek E, Mísař Z (1998) Prograde eclogite in the Gföhl Nappe, Czech Republic: new evidence on Variscan high-pressure metamorphism. J Metamorph Geol 16: 563-576

MerLet C (1994) An accurate computer correction program for quantitative electron probe microanalyses. Microchim Acta 114-115: 363-376

Michard A (1989) Rare earth element systematics in hydrothermal fluids. Geochim Cosmochim Acta 53: 745-750

Michard A, Albarède F (1986) The REE content of some hydrothermal fluids. Chem Geol 55: 51-60 
Middemost EAK (1994) Naming materials in the magma/ igneous rock system. Earth Sci Rev 37: 215-224

Mielke P, Winkler HGF (1979) Eine bessere Berechnung der Mesonorm fur granitische Gesteine. Neu Jb Mineral, Mh 1979: 471-480.

Mills RA, Elderfield H (1995) Rare earth element geochemistry of hydrothermal deposits from the active TAG mound, $26^{\circ} \mathrm{N}$ mid-Atlantic Ridge. Geochim Cosmochim Acta 59: 3511-3524

Morimoto N, Fabries J, Ferguson AK, Ginzburg IV, Ross M, Seifert FA, Zussman J, Aoki K, Gottardi G (1988) Nomenclature of pyroxenes. Mineral Mag 52: 535-550

Moyen JF (2009) High Sr/Y and La/Yb ratios: the meaning of the "adakitic signature". Lithos 112: 556-574

MuKhopadHyAy B (1991) Garnet-clinopyroxene geobarometry. The problems, prospects and an approximate solution with some applications. Amer Miner 76: 512-529

NĚMEC D (1963a) Eruptivgesteine in westmährischen Skarnen und ihre genetische Stellung. Neu Jb Mineral, Abh 100: 203-224

NĚMEC D (1963b) Mg-Skarne des westmährischen Kristallins. Sitz-Ber Akad Wiss math-naturwiss KI 172: 263-308

NĚMEC D (1968) Die Stellung des Amphibols in der Mineralparagenese westmährischer Skarngesteine. Tschermaks Mineral Petrogr Mitt 12: 321-349

NĚMEC D (1970) Chemische Zusammensetzung der CaAmphibole aus den regionalmetamorphen Skarnen Westmährens. Neu Jb Mineral, Abh 113: 50-67

NĚMEC D (1991) Regional typization of the iron skarns of the Bohemian-Moravian Heights. Acta Mus Moraviae, Sci Nat 76: 51-82

NĚMEC D (1993) Some exotic mineral assemblages of West-Moravian pegmatites. Acta Mus Moraviae, Sci Nat 78: 13-19

Newton RC, Charlu TV, Kleppa OJ (1980) Thermochemistry of the high structural state plagioclases. Geochim Cosmochim Acta 44: 933-941

NovÁk M (2005) Granitic pegmatites of the Bohemian Massif (Czech Republic); mineralogical, geochemical and regional classification and geological significance. Acta Mus Moraviae, Sci Geol 90: 3-74 (in Czech with English abstract)

Novák M, ŠKoda R, Gadas P, KRMíčEK L, ČERnÝ P (2012) Contrasting origins of the mixed signature in granitic pegmatites; examples from the Moldanubian Zone, Czech Republic. Canad Mineral 50: 1077-1094

Novák M, Kadlec T, Gadas P (2013) Geological position, mineral assemblages and contamination of granitic pegmatites in the Moldanubian Zone, Czech Republic; examples from the Vlastějovice region. J Geosci 58: 21-47

PATtison DRM (1991) Infiltration-driven anatexis in granulite facies metagabbro, Grenville Province, Ontario, Canada. J Metamorph Geol 9: 315-332
Peacock SM, Rushmer T, Thompson AB (1994) Partial melting of subducting oceanic crust. Earth Planet Sci Lett 121: 227-244

Pertold Z, Pertoldová J, Pudilová M (1997) Metamorphic history of skarns in the Gföhl Unit, Moldanubicum, Bohemian Massif, and implications for their origin. Acta Univ Carol, Geol 41: 157-166

Pertoldová J, Týcová P, Verner K, Košuličová M, Pertold Z, Košler J, Konopásek J, Pudilová M (2009) Metamorphic history of skarns, origin of their protolith and implications for genetic interpretation: an example from three units of the Bohemian Massif. J Geosci 54: 101-134

Pertoldová J, Košuličová M, Verner K, ŽáčKová E, Pertold Z, Konopásek J, Veselovský F, KošLer J (2014) Geochronology and petrology of pyroxene-garnet skarns (eastern Bohemian Massif): implications for the source and evolution of the Variscan continental crust. J Geosci 59: 367-388

Poli S (1993) The amphibolite-eclogite transformation, an experimental study on basalt. Amer J Sci 293: 1061-1107

Poli S, Schmidt MW (1995) $\mathrm{H}_{2} \mathrm{O}$ transport and release in subduction zones: experimental constraints on basaltic and andesitic systems. J Geophys Res 100: 22299-22314

Poli S, Schmidt MW (2004) Experimental subsolidus studies on epidote minerals. In: Liebscher A, Franz G (eds) Epidotes. Mineralogical Society of America Reviews in Mineralogy and Geochemistry 56: 171-195

Qian Q, Hermann J (2013) Partial melting of lower crust at 10-15 kbar: constraints on adakite and TTG formation. Contrib Mineral Petrol 165: 1195-1224

RAPP RP, WATSON EB (1995) Dehydration melting of metabasalt at 8-32 kbar: implications for continental growth and crust-mantle recycling. J Petrol 36: 891-931

ReIners PW (1998) Reactive melt transport in the mantle and geochemical signatures of mantle-derived magmas. J Petrol 39: 1039-1061

Rushmer T (1991) Partial melting of two amphibolites: contrasting experimental results under fluid-absent conditions. Contrib Mineral Petrol 107: 41-59

SAWYER EW (1991) Disequilibrium melting and the rate of melt-residuum separation during migmatization of mafic rocks from the Grenville Front, Quebec. J Petrol 32: 701-738

Schulmann K, Kröner A, Hegner E, Wendt I, Konopásek J, LEXA O, ŠTíPSKÁ P (2005) Chronological constraints on the pre-orogenic history, burial and exhumation of deep seated rocks along the eastern margin of the Variscan Orogen, Bohemian Massif, Czech Republic. Amer J Sci 305: 407-448

Schulmann K, Lexa O, Štípská P, Racek M, TajČmanová L, Konopásek J, Edel JB, Peschler A, Lehmann J (2008) Vertical extrusion and horizontal channel flow of orogenic lower crust: key exhumation mechanisms in large hot orogens? J Metamorph Geol 26: 273-297 
Simakov SK (2008) Garnet-clinopyroxene and clinopyroxene geothermobarometry of deep mantle and crust eclogites and peridotites. Lithos 106: 125-136

SkJerlie K P, Johnston AD (1992) Vapor-absent melting at $10 \mathrm{kbar}$ of a biotite- and amphibole-bearing tonalitic gneiss: implications for the generation of A-type granites. Geology 20: 263-266.

SLAVík F (1951) Skarn and its resorption of pegmatite-aplite at Dolní Líšná near Nové Město. Sbor Ústř Úst Geol 18: 573-579 (in Czech with English abstract)

STANĚK J (1977) Pegmatites from skarn near Líšná, western Moravia. Acta Univ Carol, Geol 1-2: 145-149

SuwA K, ENAMi M, Horiuchi T (1987) Chlorine-rich potassium hastingsite from West Ongul Island, Lützow-Holm Bay, East Antarctica. Mineral Mag 51: 709-714

ŠTíPSKÁ P, Powell R (2005) Constraining the P-T path of a MORB-type eclogite using pseudosections, garnet zoning and garnet-clinopyroxene thermometry: an example from the Bohemian Massif. J Metamorph Geol 23: 725-743

Tajčmanová L, Konopásek J, Schulmann K (2006) Thermal evolution of the orogenic lower crust during exhumation within a thickened Moldanubian root of the Variscan belt of Central Europe. J Metamorph Geol 24: 119-134

Vielzeuf D, Schmidt MW (2001) Melting relations in hydrous systems revisited: application to metapelites, metagreywackes and metabasalts. Contrib Mineral Petrol 141: 251-267

VRÁNA S (1987) Garnet-fassaitic pyroxene skarn from the granulite complex of southern Bohemia. Věst Ústř Úst Geol 62: 193-206

Watkins JM, Clemens JD, Treloar PJ (2007) Archaean TTGs as sources of younger granitic magmas: melting of sodic metatonalites at 0.6-1.2 GPa. Contrib Mineral Petrol 154: 91-110

WATSON EB, HARRISON TM (1983) Zircon saturation revisited: temperature and composition effects in a variety of crustal magma types. Earth Planet Sci Lett 64: 295-304
White RW, Powell R, Holland TJB (2007) Progress relating to calculation of partial melting equilibria for metapelites. J Metamorph Geol 25: 511-527

Whitney DL, Evans BW (2010) Abbreviations for names of rock forming minerals. Amer Miner 95: 185-187

WinTHER KT (1996) An experimentally based model for the origin of tonalitic and trondhjemitic melts. Chem Geol 127: 43-59

Wolf MB, Wyllie PJ (1994) Dehydration-melting of amphibolite at $10 \mathrm{kbar}$ : the effects of temperature and time. Contrib Mineral Petrol 115: 369-383

Wones DR (1989) Significance of the assemblage titanite + magnetite + quartz in granitic magmas. Amer Miner 74: 744-749

Wyllie PJ, Wolf MB (1993) Amphibolite dehydrationmelting: sorting out the solidus. In: Prichard HM, Alabaster T, Harris NBW, Neary CR (eds) Magmatic Processes and Plate Tectonics. Geological Society of London Special Publications 76, pp 405-416

Xiong XL, Adam J, Green TH (2005) Rutile stability and rutile/melt HFSE partitioning during partial melting of hydrous basalt: implications for TTG genesis. Chem Geol 218: 339-359

XIONG XL, XIA B, XU JF, Niu HC XIAO WS (2006) Na depletion in modern adakites via melt $/+6$ rock reaction within the sub-arc mantle. Chem Geol 219: 273-292

YODER HS, JR (1968) Albite-anorthite-quartz-water at 5 kb. Carnegie Inst Wash Yearb 66: 477-478

Zhang ZM, Dong X, Xiang H, He Z, Liou JG (2014) Metagabbros of the Gangdese arc root, south Tibet: implications for the growth of continental crust. Geochim Cosmochim Acta 14: 3 268-284

Ž́ÁČEK V (1997) Compositional evolution of garnet in the regionally metamorphosed Moldanubian skarn, Vlastějovice, Bohemia - evidence of the preservation of early stages pre-dating regional metamorphism. Bull Czech Geol Surv 72: 37-48

ŽÁČEK K (2007) Potassian hastingsite and potassic hastingsite from garnet-hedenbergite skarn at Vlastějovice, Czech Republic. Neu Jb Mineral, Abh 184: 161-168 